\title{
Synthesis and antimicrobial evaluation of some novel 1,2,4-triazole and 1,3,4-thiadiazole derivatives
}

\author{
Lukasz Popiołek • Urszula Kosikowska • \\ Liliana Mazur • Maria Dobosz • Anna Malm
}

Received: 12 March 2012/Accepted: 24 October 2012/Published online: 11 November 2012

(C) The Author(s) 2012. This article is published with open access at Springerlink.com

\begin{abstract}
This study presents the synthesis and spectral analysis of new derivatives of 1,2,4-triazole-3-thione and 1,3, 4-thiadiazole. New compounds were prepared by cyclization reaction of acyl thiosemicarbazide derivatives in the presence of alkaline and acidic media. All synthesized compounds were screened for their in vitro antibacterial activity by using the agar dilution technique. Six of the compounds had potential activity against Gram-positive bacteria (minimal inhibitory concentration $[\mathrm{MIC}]=15.63-500 \mu \mathrm{g} / \mathrm{mL}$ ). Some compounds showed good activity especially against Bacillus subtilis ATCC 6633 (MIC $=15.63-250 \mu \mathrm{g} / \mathrm{mL})$, Staphylococcus aureus ATCC 25923 (MIC $=31.25-250 \mu \mathrm{g} / \mathrm{mL}$ ), and Micrococcus luteus ATCC $10240(\mathrm{MIC}=125-250 \mu \mathrm{g} / \mathrm{mL})$.
\end{abstract}

Keywords Antimicrobial activity .

1,2,4-Triazole derivatives $\cdot 1,3,4$-Thiadiazole derivatives

\section{Introduction}

For the last few decades, there has been a tremendous growth of research in the synthesis of nitrogen and sulfur containing heterocyclic derivatives because of their utility

Ł. Popiołek $(\bowtie) \cdot$ M. Dobosz

Department of Organic Chemistry, Faculty of Pharmacy,

Medical University, Lublin, Poland

e-mail: lukasz.popiolek@umlub.pl

U. Kosikowska · A. Malm

Department of Pharmaceutical Microbiology, Faculty

of Pharmacy, Medical University, Lublin, Poland

\section{Mazur}

Department of General and Coordination Chemistry, Faculty

of Chemistry, Maria Curie-Sklodowska University,

Lublin, Poland in various applications, such as pharmaceuticals, propellants, explosives, and pyrotechnics.

The recent literature is enriched with progressive findings about the synthesis and pharmacological action of triazole and thiadiazole derivatives. Heterocycles bearing 1,2,4-triazole and 1,3,4-thiadiazole moiety are reported to show a broad spectrum of biologic activity such as analgesic (Turan-Zitouni et al., 1999), antiphlogistic (Harish et al., 2008; El Shehry et al., 2010; Schenone et al., 2006), anticonvulsant (Dogan et al., 2002; Almasirad et al., 2004), antitumor (Duran et al., 2002; Kumar et al., 2010), antiviral (Al-Soud et al., 2004), antifungal (Collin et al., 2003; Wei et al., 2006), antibacterial (Ulusoy et al., 2001; Gülerman et al., 2001; Padmavathi et al., 2009; Demirbas et al., 2009; Liesen et al., 2010), and antitubercular action (Klimešová et al., 2004; Gadad et al., 2004; Shiradkar et al., 2007). A large number of ring systems containing triazoles and thiadiazoles have been incorporated into a wide variety of therapeutically interesting drug candidates. Some of them are approved as drugs, for example, alprazolam (Pick, 1997), etizolam (Shiroki et al., 1976), or vibunazole (Holmwood et al., 1982). Vorozole, letrozole, and anastrozole are non-steroidal drugs used for the treatment of cancer (Clemons et al., 2004). Triazoles are also used as intermediates for the synthesis of antifungal agents such as fluconazole, voriconazole, and itraconazole (Bailey et al., 1990; McGinnis et al., 1997).

In continuation of our research program on the synthesis of 1,2,4-triazole and 1,3,4-thiadiazole compounds exhibiting biologic activity, it was thought to be interesting to synthesize new antimicrobial agents, especially when the development of resistance of pathogenic bacteria toward available antibiotics is rapidly becoming a major worldwide problem. The designing of new compounds to deal with resistant bacteria has become one of the most 
important areas of antibacterial research today. In addition, primary and opportunistic microbial infections continue to increase rapidly because of the increased number of immunocompromised patients.

Keeping in mind the above facts, we designed and synthesized series of some new 1,2,4-triazole-3-thione and 1,3,4-thiadiazole derivatives and evaluated their in vitro antibacterial activity.

\section{Results and discussion}

\section{Chemistry}

The substituted 1,2,4-triazole and 1,3,4-thiadiazole derivatives are generally obtained by the cyclization reaction of thiosemicarbazide derivatives, which is dependent not only on the $\mathrm{pH}$ of the medium, but also on the nature of substituents in thiosemicarbazide derivatives (Dobosz and Pachuta-Stec, 1995, 1996). The presence of alkaline media usually promotes the reaction of cyclization to obtain 1,2, 4-triazole systems, whereas in acidic media, 1,3,4-thiadiazole derivatives were obtained.

4,5-Diphenyl-4H-1,2,4-triazole-3-thione 1 was a starting material for the synthesis of new compounds, which consist of two 1,2,4-triazole systems or 1,2,4-triazole and 1,3, 4-thiadiazole systems connected with the S-methylene group. Compound $\mathbf{1}$ was obtained by the cyclization reaction of 1,4-diphenyl thiosemicarbazide in alkaline media. In the next step, compound 1, which can exist in two tautomeric forms, was submitted to the reaction with ethyl bromoacetate in the presence of sodium ethanolate. The reaction let us obtain ethyl 2-[(4,5-diphenyl-4H-1,2,4triazol-3-yl)sulfanyl] acetate (2). The direction of this reaction to form a thio derivative of compound $\mathbf{1}$ was revealed and confirmed by X-ray crystallography (Dobosz et al., 1996). The mechanism of this reaction as a nucleophilic substitution on the sulfur atom had been studied and investigated earlier (Wujec and Paneth, 2007).

Subsequently, compound $\mathbf{2}$ was converted to hydrazide 3 in reaction with $100 \%$ hydrazine hydrate. Then, reactions of hydrazide $\mathbf{3}$ with various isothiocyanates were performed in two ways.

All new thiosemicarbazide derivatives $\mathbf{4 a}-\mathbf{l}$ were obtained by heating reactants in an oil bath; temperatures were selected experimentally $\left(t=50-110^{\circ} \mathrm{C}\right)$. Thiosemicarbazide derivatives $\mathbf{4 a}, \mathbf{c}, \mathbf{d}$ were products of the reaction of hydrazide $\mathbf{3}$ with appropriate isothiocyanates in the presence of diethyl ether carried in room temperature.

A new group of compounds, which consist of two 1,2, 4-triazole-3-thione derivatives $\mathbf{5 a}-\mathbf{i}$, were acquired in cyclization reaction with $2 \%$ aqueous solution of sodium hydroxide of new acyl thiosemicarbazide derivatives $\mathbf{4 a}-\mathbf{i}$.
In three cases, the cyclization reaction of thiosemicarbazide derivatives $\mathbf{4 j} \mathbf{j}-\mathbf{l}$ in alkaline media was accompanied by hydrolysis. The [(4,5-diphenyl-4H-1,2,4-triazol-3-yl)sulfanyl] acetic acid $\mathbf{8}$ was obtained in cyclization of 4-ethoxycarbonyl-1-substituted thiosemicarbazide $\mathbf{4 j}$. This compound was described earlier, but it was obtained in a different way (Kaplaushenko et al., 2008). The cyclization in alkaline media of the thiosemicarbazide which contains the ethoxycarbonylmethyl group $\mathbf{4 k}$ and benzoyl $\mathbf{4 l}$ in the fourth position led us to obtain substituted 1,2,4-triazole-3-thione derivatives 9, 10. These compounds were subjected to the reaction with pyrrolidine and formaldehyde to get new $N$-substituted 1,2,4-triazole-3-thione derivatives 11, 12.

The thiosemicarbazide derivatives $\mathbf{4 a - i}$ were also submitted to the cyclization reaction in acidic media. In this way, we were able to obtain new compounds which consist of 1,2,4-triazole-3-thione and 1,3,4-thiadiazole system, that is (5-aminosubstituted)-2-\{[(4,5-diphenyl-4H-1,2,4-triazol3-yl)sulfanyl]methyl\}-1,3,4-thiadiazole 6a-i. Afterward, the derivatives of $N, N$-disubstituted acetamide $7 \mathbf{a}-\mathbf{i}$ were obtained by the acylation reaction of 2,5-disubstituted1,3,4-thiadiazoles $\mathbf{6 a - i}$ with acetic anhydride.

The mechanism of cyclization of thiosemicarbazide was investigated earlier (Siwek and Paneth, 2007). It was proved that the direction of cyclization is dependent on the nature of substituents and acidic or alkaline media (Siwek et al., 2010).

The structure of all obtained compounds was confirmed by elementary analysis, IR and ${ }^{1} \mathrm{H}$ NMR spectra. Some of the compounds were also submitted to ${ }^{13} \mathrm{C}$ NMR and MS spectra analyses. The crystal structure of the representative compound 2 was determined by the single-crystal X-ray analysis. The reactions were performed according to Schemes 1 and 2.

In the IR spectra of the thiosemicarbazide derivatives $\mathbf{4 a}-\mathbf{l}$, the following characteristic absorption bands were observed: about $1,700 \mathrm{~cm}^{-1}$ corresponding to the $\mathrm{C}=\mathrm{O}$ group and in the range of $1,300 \mathrm{~cm}^{-1}$ corresponding to the $\mathrm{C}=\mathrm{S}$ group. Compounds which consist of two 1,2,4-triazole systems 5a-i, 9, 10 had absorption bands: about $1,300 \mathrm{~cm}^{-1}$ ( $\mathrm{C}=\mathrm{S}$ group), about $1,500 \mathrm{~cm}^{-1}$ (C-N group), in the range of $1,600 \mathrm{~cm}^{-1}(\mathrm{C}=\mathrm{N}$ group), and about $3,100-3,200 \mathrm{~cm}^{-1}$ (NH group). Then, in the IR spectra of the new derivatives of 1,3,4-thiadiazole $\mathbf{6 a}-\mathbf{i}$, the following characteristic absorption bands were observed: in the range of $1,500 \mathrm{~cm}^{-1}$ corresponding to the $\mathrm{C}-\mathrm{N}$ group and in the range of $1,600 \mathrm{~cm}^{-1}$ corresponding to the $\mathrm{C}=\mathrm{N}$ group and about 3,200 $\mathrm{cm}^{-1}$ for the NH group. Compounds $7 \mathbf{a}-\mathbf{i}, 11$ had a characteristic absorption band at about $1,700 \mathrm{~cm}^{-1}$ for the $\mathrm{C}=\mathrm{O}$ group.

${ }^{1} \mathrm{H}$ NMR spectra of the thiosemicarbazide derivatives 4a-l show three proton signals typical for the NH group in the $\delta 8.32-12.87 \mathrm{ppm}$ range, whereas for the new compounds consisting of two 1,2,4-triazole system 5a-i, 9, 10, 

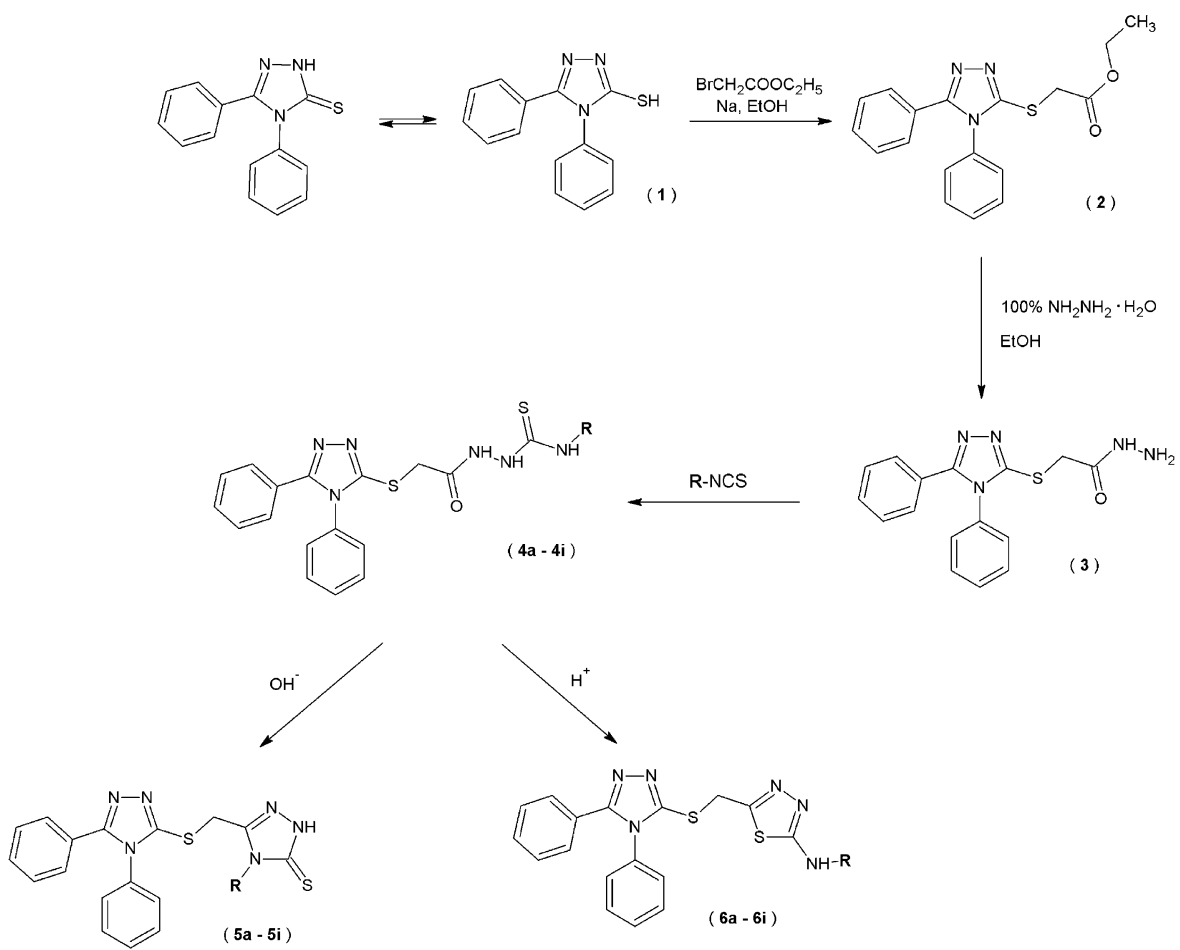

$\left(\mathrm{CH}_{3} \mathrm{CO}\right)_{2} \mathrm{O}$

$\mathrm{CH}_{3} \mathrm{COOH}$

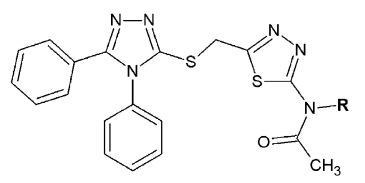

$(7 a-7 i)$

R: a: $\mathrm{C}_{2} \mathrm{H}_{5}$, b: $\mathrm{CH}_{2} \mathrm{CH}=\mathrm{CH}_{2}$, c: $\mathrm{C}_{6} \mathrm{H}_{11}$, d: $\mathrm{C}_{6} \mathrm{H}_{5}$, e: $4-\mathrm{BrC}_{6} \mathrm{H}_{4}$, f: $4-\mathrm{ClC}_{6} \mathrm{H}_{4}$, g: $4-\mathrm{CH}_{3} O \mathrm{OC}_{6} \mathrm{H}_{4}$, h: $\mathrm{CH}_{2} \mathrm{C}_{6} \mathrm{H}_{5}$, i: $4-\mathrm{CH}_{3} \mathrm{OC}_{6} \mathrm{H}_{4} \mathrm{CH}_{2}$

Scheme 1 Synthesis of new derivatives of thiosemicabrazide, 1,2,4-triazole-3-thione and 1,3,4-thiadiazole

one proton signal of the $\mathrm{NH}$ group was observed in the $\delta 13.62-14.13 \mathrm{ppm}$ range. The 1,3,4-thiadiazole derivatives $\mathbf{6 a}-\mathbf{i}$ had one typical proton signal of the $\mathrm{NH}$ group in the $\delta$ 9.35-10.47 ppm range. Derivatives of $N, N$-disubstituted acetamide 7a-i had one proton signal of the $\mathrm{CH}_{3}$ group in the $\delta 2.06-2.16 \mathrm{ppm}$ range. Compound $\mathbf{1 1}$ had one proton signal for the $\mathrm{OH}$ group $(\delta 13.68 \mathrm{ppm})$ and for the pyrrolidine substituent. Similarly, 4,5-disubstituted-2(pyrrolidin-1-ylmethyl)-1,2,4-triazole-3-thione $\mathbf{1 2}$ had one typical proton signal for the NH group $(\delta 14.68 \mathrm{ppm})$ and for the pyrrolidine substituent.

Compound 2 crystallizes in the monoclinic space group $P 2_{1} /$ $n$ with one molecule in the asymmetric unit of the crystal. The diffraction study confirmed that the molecule contained the 1,2,4-triazole ring, substituted at $\mathrm{C} 3, \mathrm{~N} 4$, and $\mathrm{C} 5$ atoms by thioacetate moiety and two phenyl rings, respectively (Fig. 1). The chain of atoms from S1 to ethyl $\mathrm{C} 4$ is almost planar $($ rmsd $=0.006 \AA)$; a higher twist $\left(4.56^{\circ}\right)$ is observed around the $\mathrm{C} 4-\mathrm{O} 1$ bond in the solid state. The best plane of the atoms of thioacetate unit intersects that of the 1,2,4-triazole ring at the angle of $81.4(1)^{\circ}$. The carbonyl $\mathrm{C} 2=\mathrm{O} 2$ group in 2 is cis oriented with respect to the thioether S1 atom. What is more, it seems to be preferred in thioacetate derivatives in the solid state (CSD, V.5.33, Allen, 2002). The geometric parameters of the ester group are within normal ranges (International Tables for Crystallography, 1995). Likewise, the S1-C3 and S1-C1 distances, being of 1.738(2) and 1.789(3) $\AA$, are in agreement with the single thioether $\mathrm{C}-\mathrm{S}$ bonds. The most characteristic feature of the crystal of $\mathbf{2}$ is the presence of centrosymmetric molecular dimers. The "head-to-head" oriented molecules within the dimer form short $\mathrm{S} 1 \cdots \mathrm{O} 2^{\mathrm{i}}$ [3.268(3) $\AA$; (i) $1-x,-y,-z$ ] contacts which might be attractive in their nature (Ramasubbu and Parthasarathy, 1989).

\section{Microbiology}

On the basis of the preliminary results obtained by the agar dilution method, it was shown that some of the newly 


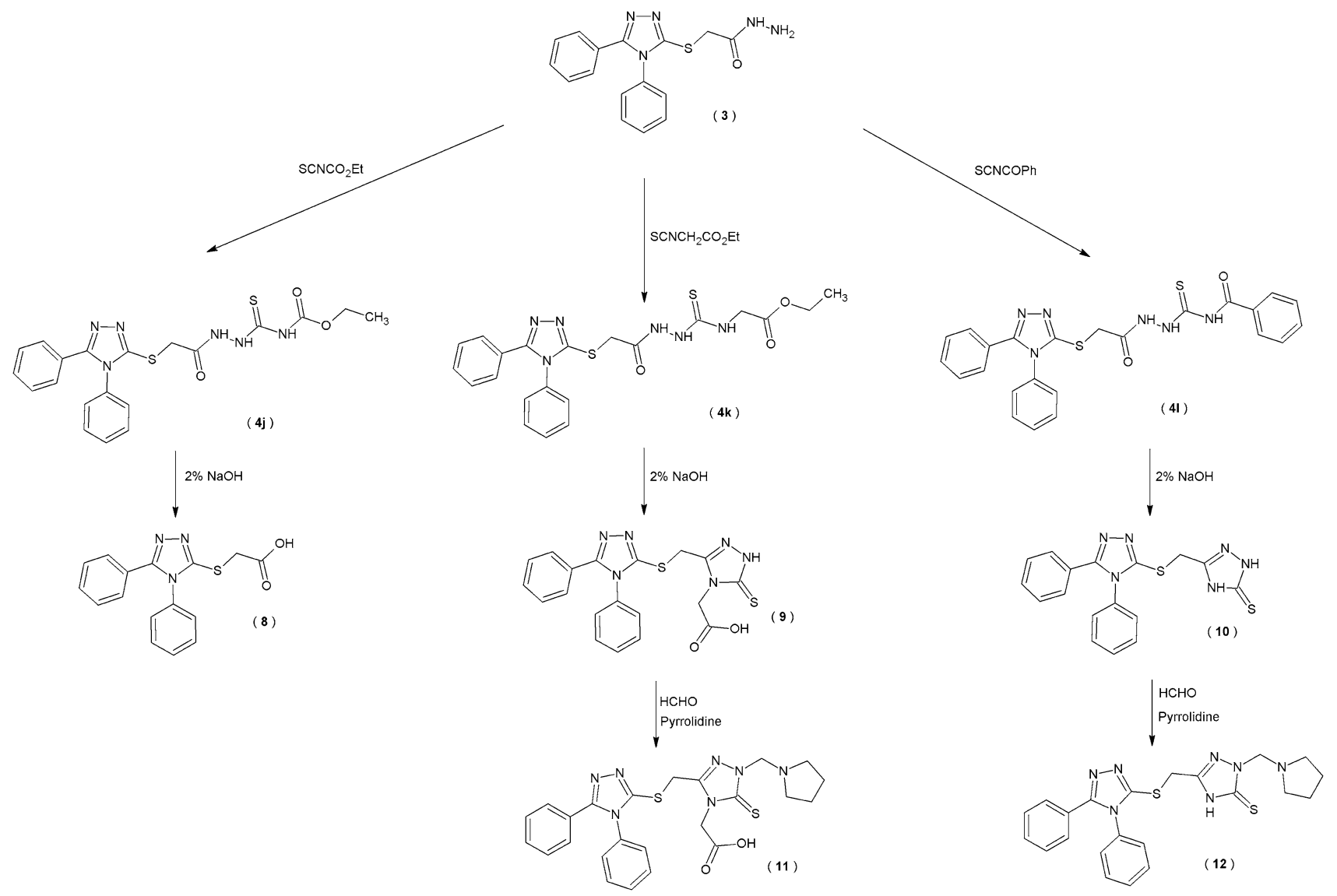

Scheme 2 Synthesis of new derivatives of 1,2,4-triazole-3-thione

synthesized compounds had the potential activity against reference strains of Gram-positive bacteria. None of the compounds had inhibitory effect on the Gram-negative bacteria growth.

According to Table 1, on the basis of minimal inhibitory concentration (MIC) values obtained by the broth microdilution method, it was shown that the highest activity had compound 41 with $\mathrm{MIC}=31.25 \mu \mathrm{g} / \mathrm{mL}$ against Staphylococcus aureus ATCC 25923, MIC $=125 \mu \mathrm{g} / \mathrm{mL}$ against Staphylococcus epidermidis ATCC 12228, Bacillus cereus ATCC 10876, and Micrococcus luteus ATCC 10240 or MIC $=250 \mu \mathrm{g} / \mathrm{mL}$ against S. aureus ATCC 6538 and Bacillus subtilis ATCC 6633. Compound $\mathbf{6 h}$ was also active especially against $B$. subtilis ATCC 6633 with $\mathrm{MIC}=15.63 \mu \mathrm{g} / \mathrm{mL}$ and with MIC $=125 \mu \mathrm{g} / \mathrm{mL}$ against $M$. luteus ATCC 10240 or MIC $=250 \mu \mathrm{g} / \mathrm{mL}$ against $S$. aureus ATCC 25923.

The somewhat lower activity against reference strains of Gram-positive bacteria was shown by compound 5c (MIC values from 250 to $1,000 \mu \mathrm{g} / \mathrm{mL}$ ). According to our results, MICs of cefuroxime, which has been extensively used to treat bacterial infections, were $0.24-1.95 \mu \mathrm{g} / \mathrm{mL}$ for Staphylococcus species and $0.49-62.5 \mu \mathrm{g} / \mathrm{mL}$ for the other Gram-positive bacteria.

With our research, it has been established that the introduction of the benzoyl group in thiosemicarbazide and the benzyl group in 1,3,4-thiadiazole derivative yielded active compounds endowed with a wide spectrum of antimicrobial activities.

The compounds $\mathbf{4 l}$ and $\mathbf{6 h}$ with potential activity against the reference strains of Gram-positive bacteria may be regarded as precursor compounds for searching for new derivatives showing antimicrobial activity against pathogenic (e.g. S. aureus) or opportunistic (e.g. S. epidermidis, M. luteus, B. subtilis, or B. cereus) bacteria.

\section{Experimental}

\section{Chemistry}

Melting points were determined in Fisher-Johns blocks (Pittsburgh, US) and presented without any corrections. The IR spectra $\left(v, \mathrm{~cm}^{-1}\right)$ were recorded in $\mathrm{KBr}$ tablets using a Specord IR-75 spectrophotometer (Germany). The NMR spectra were recorded on a Bruker Avance 300 apparatus (Bruker BioSpin GmbH, Rheinstetten/Karlsruhe, Germany) in dimethyl sulfoxide (DMSO)- $d_{6}$ with TMS as the internal standard, and chemical shifts are given in ppm ( $\delta$-scale). The MS spectra were recorded on a Thermo- 
Fig. 1 Molecular structure of 2 with atom-labeling scheme. Displacement ellipsoids are drawn at the $50 \%$ probability level. Selected bond distances (A): C3-S1 1.738(2), C1-S1 1.789(3), C1-C2 1.494(4), C2-O1 1.321(3), C2-O2 1.191(3), C4-O1 1.460(3)
Table 1 The inhibitory activities of newly synthesized compounds against Grampositive bacteria on the basis of MIC $(\mu \mathrm{g} / \mathrm{mL})$ values determined by broth microdilution method

Bold values indicate the lowest MIC nd Not determined,

Sa25923 S. aureus ATCC 25923, Sa6538 S. aureus ATCC 6538, Se12228 S. epidermidis ATCC 12228, Bs6633 B. subtilis ATCC 6633, Bc10876

B. cereus ATCC 10876 , Ml10240 M. luteus ATCC 10240

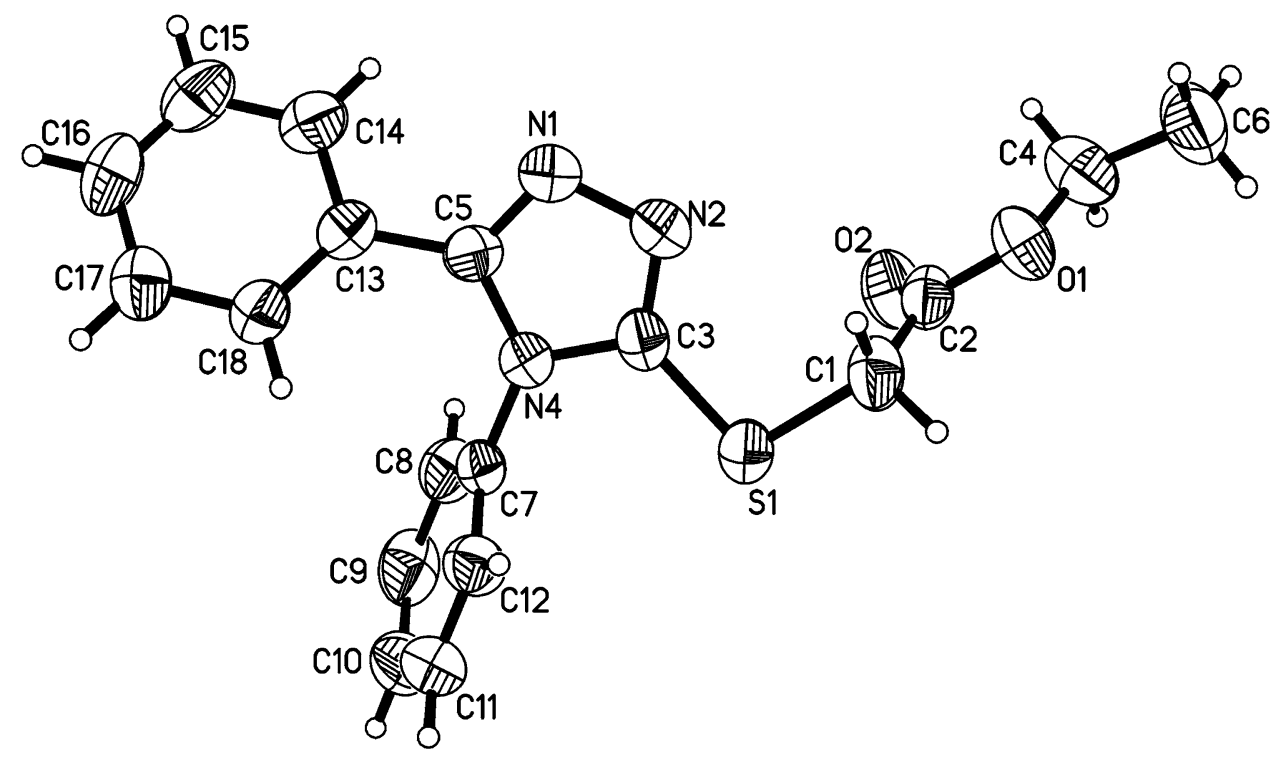

Finnigan Trace DSQ GC MS apparatus (Waltham, Massachusetts, US). Chemicals were purchased from Merck Co., or Lancaster and used without further purification.

The purity of the obtained compounds was checked by TLC on aluminum oxide $60 \mathrm{~F}_{254}$ plates (Merck Co., Whitehouse Station, New Jersey, US), in a $\mathrm{CHCl}_{3} / \mathrm{C}_{2} \mathrm{H}_{5} \mathrm{OH}$ (10:1, $\mathrm{v} / \mathrm{v})$ solvent system with UV visualization $(\lambda=254 \mathrm{~nm})$.

Elemental analysis of the obtained compounds was performed for $\mathrm{C}, \mathrm{H}, \mathrm{N}, \mathrm{S}$. The maximum percentage differences between calculated and found values for each element were within the error and amounted to $\pm 0.4 \%$.

\section{Crystal data for 2}

$\mathrm{C}_{18} \mathrm{H}_{17} \mathrm{~N}_{3} \mathrm{O}_{2} \mathrm{~S}$, colorless prism, $0.45 \times 0.29 \times 0.14 \mathrm{~mm}^{3}$, monoclinic, $P 2_{1} / n, \quad a=11.692(1) \AA, \quad b=9.414(1) \AA$, $c=15.740(2) \AA, \beta=100.24(1)^{\circ}, V=1,704.9(3) \AA^{3}, Z=4$, $d_{\text {calc }}=1.322 \mathrm{~g} \mathrm{~cm}^{-3}, \mu=0.205 \mathrm{~mm}^{-1}, \mathrm{GooF}=0.977$, data/ restraints/parameters 3930/0/217 $\left(R_{\text {int }}=0.04\right)$, final $R$ indices $(I>2 \sigma(I)): R_{1}=0.0548, w R_{2}=0.0888, R$ indices (all data): $R_{1}=0.1867, w R_{2}=0.1202$, largest diff. peak and hole: 0.16

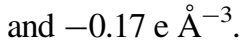

Single-crystal diffraction data were measured at room temperature on an Oxford Diffraction Xcalibur diffractometer with the graphite-monochromated Mo $\mathrm{K} \alpha$ radiation $(\lambda=0.71073)$. The programs CrysAlis CCD and CrysAlis Red (Oxford Diffraction, Xcalibur CCD System, 2006) were used for data collection, cell refinement, and data reduction. The intensity data were corrected for Lorentz and polarization effects. The structure was solved by direct methods using SHELXS-97 and refined by the full-matrix least-squares on $F^{2}$ using the SHELXL-97 (Sheldrick, 2008). All non-hydrogen atoms were refined with anisotropic displacement parameters. All $\mathrm{H}$-atoms were positioned geometrically and allowed to ride on their parent atoms with $U_{\text {iso }}(\mathrm{H})=1.2 U_{\mathrm{eq}}(\mathrm{C})$. 
Crystallographic data have been deposited with the CCDC, 12 Union Road, Cambridge, CB2 1EZ, UK (fax: +44 1223 366033; e-mail: deposit@ccdc.cam.ac.uk or http://www.ccdc.cam.ac.uk) and are available on request, quoting the deposition number CCDC 860357.

Ethyl 2-[(4,5-diphenyl-4H-1,2,4-triazol-3-yl)

sulfanyl]acetate (2)

\section{Method A}

$0.23 \mathrm{~g}$ (10 mmol) of sodium was added to $5 \mathrm{~mL}$ of anhydrous ethanol. The solution was placed in a three-necked flask equipped with reflux condenser and closed with a tube of $\mathrm{CaCl}_{2}$ and mercury stirred. The content was mixed till the sodium dissolved completely and then $2.53 \mathrm{~g}(10 \mathrm{mmol})$ of 4,5-diphenyl-4H-1,2,4-triazole-3-thione (1) was added. Then, $1.22 \mathrm{~mL}$ ethyl bromoacetate was added drop by drop. The content of the flask was mixed for $4 \mathrm{~h}$ and left at room temperature for $12 \mathrm{~h}$. Then, $10 \mathrm{~mL}$ of anhydrous ethanol was added and heated for $1 \mathrm{~h}$. The mixture was filtered of inorganic compounds. After cooling, the precipitate was filtered and crystallized from ethanol.

\section{Method B}

$2.53 \mathrm{~g}$ (10 mmol) of 4,5-diphenyl-4H-1,2,4-triazole-3thione (1) was dissolved in $10 \mathrm{~mL}$ of $N, N$-dimethylformamide. Then, $1 \mathrm{~g}$ of potassium carbonate and $1.22 \mathrm{~mL}$ of ethyl bromoacetate were added to the solution. The content of the flask was refluxed for $2 \mathrm{~h}$. The mixture was filtered of inorganic compounds. Then, the distilled water was added and the precipitated compound was filtered, dried, and crystallized from ethanol.

Yield: $67.8 \%$, mp: $92-94{ }^{\circ} \mathrm{C}$ (dec.). Analysis for $\mathrm{C}_{18} \mathrm{H}_{17} \mathrm{~N}_{3} \mathrm{O}_{2} \mathrm{~S}$ (339.41); calculated: C, 63.70; H, 5.05; N, 12.38; S, 9.45; found: C, 63.92; H, 5.03; N, 12.41; S, 9.48. IR (KBr), $v\left(\mathrm{~cm}^{-1}\right): 3091(\mathrm{CH}$ aromatic), 2955, $1422(\mathrm{CH}$ aliphatic), $1701(\mathrm{C}=\mathrm{O}), 1611(\mathrm{C}=\mathrm{N}), 676(\mathrm{C}-\mathrm{S}) .{ }^{1} \mathrm{H}$ NMR $\left(\mathrm{DMSO}-d_{6}\right) \delta(\mathrm{ppm}): 1.19\left(\mathrm{t}, J=6 \mathrm{~Hz}, 3 \mathrm{H}, \mathrm{CH}_{3}\right), 4.09$ (s, $\left.2 \mathrm{H}, \mathrm{CH}_{2}\right), 4.11-4.17$ (q, $J=5 \mathrm{~Hz}, J=5 \mathrm{~Hz}, 2 \mathrm{H}, \mathrm{CH}_{2}$ ), 7.31-7.58 (m, 10H, 10ArH).

[(4,5-Diphenyl-4H-1,2,4-triazol-3-yl)sulfanyl] acetohydrazide (3)

$0.5 \mathrm{~mL}$ of $100 \%$ hydrazine hydrate was added to $3.39 \mathrm{~g}$ (10 mmol) of compound 2 in $10 \mathrm{~mL}$ of anhydrous ethanol. The mixture was left at room temperature for $24 \mathrm{~h}$. The precipitation of hydrazide $\mathbf{3}$ was filtered, dried, and crystallized from ethanol.

Yield: $91.4 \%$, mp: $196-198{ }^{\circ} \mathrm{C}$ (dec.). Analysis for $\mathrm{C}_{16} \mathrm{H}_{15} \mathrm{~N}_{5} \mathrm{OS}$ (325.39); calculated: C, 59.06; H, 4.65; N,
21.52; S, 9.82; found: C, 59.10; H, 4.63; N, 21.49; S, 9.78. IR $(\mathrm{KBr}), v\left(\mathrm{~cm}^{-1}\right)$ : $3105(\mathrm{CH}$ aromatic), 2980, $1423(\mathrm{CH}$ aliphatic), $1698(\mathrm{C}=\mathrm{O}), 1611(\mathrm{C}=\mathrm{N}), 1522(\mathrm{C}-\mathrm{N}), 699(\mathrm{C}-\mathrm{S})$. ${ }^{1} \mathrm{H}$ NMR (DMSO- $\left.d_{6}\right) \delta(\mathrm{ppm}): 3.91\left(\mathrm{~s}, 2 \mathrm{H}, \mathrm{CH}_{2}\right), 4.31(\mathrm{~s}, 2 \mathrm{H}$, $\mathrm{NH}_{2}$ ), 7.31-7.57 (m, 10H, 10ArH), 9.40 (brs, 1H, NH).

Derivatives of thiosemicarbazide $(\mathbf{4 a}-\mathbf{l})$

General method (for compounds $\mathbf{4 a - l}$ )

A mixture of $3.25 \mathrm{~g}$ (10 mmol) of hydrazide (3) and $10 \mathrm{mmol}$ appropriate isothiocyanate was heated in an oil bath at $50-110{ }^{\circ} \mathrm{C}$ for $8-20 \mathrm{~h}$. The product was washed with diethyl ether to remove unreacted isothiocyanate. Then it was filtered, dried, and crystallized from ethanol $\mathbf{4 a}-\mathbf{c}, \mathbf{d}, \mathbf{g}-\mathbf{l}$, butanol $\mathbf{4 e}$, or methanol $4 \mathbf{f}$.

\section{Method B (for compounds $\mathbf{4 a}, \boldsymbol{c}, \boldsymbol{d})$}

$10 \mathrm{mmol}$ of appropriate isothiocyanate was added to $3.25 \mathrm{~g}$ (10 mmol) of hydrazide 3 in $10 \mathrm{~mL}$ of anhydrous diethyl ether. The mixture, placed in a conical bulb, was mixed for $5 \mathrm{~min}$ and left in room temperature for $24 \mathrm{~h}$. The precipitation of thiosemicarbazide $\mathbf{4 a}, \mathbf{c}, \mathbf{d}$ was filtered, dried, and crystallized from ethanol. The obtained compounds had the same melting points as the compounds obtained by the general method.

4-Ethyl-1-\{[(4,5-diphenyl-4H-1,2,4-triazol-3-yl)sulfanyl] acetyl\} thiosemicarbazide (4a) Yield: $94.0 \%$. Temperature of reaction: $70{ }^{\circ} \mathrm{C}$ for $8 \mathrm{~h}, \mathrm{mp}$ : $205-207{ }^{\circ} \mathrm{C}$ (dec.). Analysis for $\mathrm{C}_{19} \mathrm{H}_{20} \mathrm{~N}_{6} \mathrm{OS}_{2}$ (412.53); calculated: C, 55.32; H, 4.89; N, 20.37; S, 15.54; found: C, 55.23; H, 4.88; N, 20.43; S, 15.59. IR (KBr), $v\left(\mathrm{~cm}^{-1}\right): 3199(\mathrm{NH}), 3101(\mathrm{CH}$ aromatic), 2974, 1453, 741 ( $\mathrm{CH}$ aliphatic), 1699 (C=O), $1607(\mathrm{C}=\mathrm{N}), 1519(\mathrm{C}-\mathrm{N}), 1329(\mathrm{C}=\mathrm{S}), 691(\mathrm{C}-\mathrm{S}) .{ }^{1} \mathrm{H}$ NMR (DMSO- $\left.d_{6}\right) \delta(\mathrm{ppm}): 1.12\left(\mathrm{t}, J=9 \mathrm{~Hz}, 3 \mathrm{H}, \mathrm{CH}_{3}\right)$, $3.51-3.60$ (q, $\left.J=7.5 \mathrm{~Hz}, J=7.5 \mathrm{~Hz}, 2 \mathrm{H}, \mathrm{CH}_{2}\right), 3.90$ (s, $\left.2 \mathrm{H}, \mathrm{CH}_{2}\right), 7.34-7.57(\mathrm{~m}, 10 \mathrm{H}, 10 \mathrm{ArH}), 8.32,9.33,10.25$ (3brs, 3H, 3NH). ${ }^{13} \mathrm{C}$ NMR $\delta(\mathrm{ppm}): 14.61\left(\mathrm{CH}_{3}\right), 30.75(-$ $\left.\mathrm{S}-\mathrm{CH}_{2}-\right), 33.90\left(-\mathrm{CH}_{2}-\mathrm{CH}_{3}\right), 126.42,127.68,127.95$, 128.79, 130.07, 130.11 (10CH aromatic), 130.33, 133.65 (2C aromatic), $152.08(\mathrm{C}-\mathrm{S}), 154.59$ (C-3 triazole), 166.82 $(\mathrm{C}=\mathrm{O}), 181.23(\mathrm{C}=\mathrm{S}) . \mathrm{MS} \mathrm{m} / z(\%): 412\left(\mathrm{M}^{+}, 2\right), 397$ (3), 335 (2), 325 (5), 294 (26), 253 (61), 252 (100), 194 (21), 180 (20), 149 (20), 118 (23), 104 (25), 91 (44), 77 (79).

4-Allyl-1-\{[(4,5-diphenyl-4H-1,2,4-triazol-3-yl)sulfanyl] acetyl\} thiosemicarbazide (4b) Yield: $90.7 \%$. Temperature of reaction: $55{ }^{\circ} \mathrm{C}$ for $12 \mathrm{~h}$, mp: $192-194{ }^{\circ} \mathrm{C}$ (dec.). Analysis for $\mathrm{C}_{20} \mathrm{H}_{20} \mathrm{~N}_{6} \mathrm{OS}_{2}$ (424.54); calculated: C, 56.58; H, 4.75; N, 19.79; S, 15.10; found: C, 56.53; H, 4.76; N, 19.81; S, 15.14. IR (KBr), $v\left(\mathrm{~cm}^{-1}\right): 3218(\mathrm{NH}), 3078(\mathrm{CH}$ 
aromatic), 2963, 1431, 761 ( $\mathrm{CH}$ aliphatic), $1705(\mathrm{C}=\mathrm{O})$, $1603(\mathrm{C}=\mathrm{N}), 1511(\mathrm{C}-\mathrm{N}), 1351(\mathrm{C}=\mathrm{S}), 686(\mathrm{C}-\mathrm{S}) .{ }^{1} \mathrm{H}$ NMR (DMSO- $\left.d_{6}\right) \delta(\mathrm{ppm}): 3.95\left(\mathrm{~s}, 2 \mathrm{H}, \mathrm{CH}_{2}\right), 4.13(\mathrm{~d}$, $\left.J=5 \mathrm{~Hz}, 2 \mathrm{H}, \mathrm{CH}_{2}\right), 5.02-5.13(\mathrm{dd}, J=5 \mathrm{~Hz}, J=5 \mathrm{~Hz}$, $\left.2 \mathrm{H},=\mathrm{CH}_{2}\right), 5.79-5.90(\mathrm{~m}, 1 \mathrm{H}, \mathrm{CH}), 7.35-7.57(\mathrm{~m}, 10 \mathrm{H}$, $10 \mathrm{ArH}), 8.45,9.45,10.33$ (3brs, 3H, 3NH).

4-Cyclohexyl-1-\{[(4,5-diphenyl-4H-1,2,4-triazol-3-yl)sulfanyl]acetyl\} thiosemicarbazide (4c) Yield: $64.5 \%$. Temperature of reaction: $50{ }^{\circ} \mathrm{C}$ for $12 \mathrm{~h}, \mathrm{mp}: 188-190{ }^{\circ} \mathrm{C}$ (dec.). Analysis for $\mathrm{C}_{23} \mathrm{H}_{26} \mathrm{~N}_{6} \mathrm{OS}_{2}$ (466.62); calculated: $\mathrm{C}$, $59.20 ; \mathrm{H}, 5.62 ; \mathrm{N}, 18.01 ; \mathrm{S}, 13.74$; found: C, 59.35; H, 5.63; $\mathrm{N}, 17.95 ; \mathrm{S}, 13.70$. IR (KBr), $v\left(\mathrm{~cm}^{-1}\right): 3208(\mathrm{NH}), 3109$ (CH aromatic), 2987, 1424, 753 (CH aliphatic), 1699 $(\mathrm{C}=\mathrm{O}), 1595(\mathrm{C}=\mathrm{N}), 1519(\mathrm{C}-\mathrm{N}), 1331(\mathrm{C}=\mathrm{S}), 689(\mathrm{C}-\mathrm{S})$. ${ }^{1} \mathrm{H}$ NMR (DMSO- $\left.d_{6}\right) \delta(\mathrm{ppm}): 1.01-1.72\left(\mathrm{~m}, 10 \mathrm{H}, 5 \mathrm{CH}_{2}\right.$ cyclohexane), $3.87\left(\mathrm{~s}, 2 \mathrm{H}, \mathrm{CH}_{2}\right), 4.31(\mathrm{~m}, 1 \mathrm{H}, \mathrm{CH}$ cyclohexane), 7.28-7.56 (m, 10H, 10ArH), 8.71, 9.35, 10.20 (3brs, 3H, 3NH).

4-Phenyl-1-\{[(4,5-diphenyl-4H-1,2,4-triazol-3-yl)sulfanyl] acetyl\} thiosemicarbazide (4d) Yield: $91.0 \%$. Temperature of reaction: $50{ }^{\circ} \mathrm{C}$ for $15 \mathrm{~h}, \mathrm{mp}: 178-180{ }^{\circ} \mathrm{C}$ (dec.). Analysis for $\mathrm{C}_{23} \mathrm{H}_{20} \mathrm{~N}_{6} \mathrm{OS}_{2}$ (460.57); calculated: C, 59.98; $\mathrm{H}$, 4.38; N, 18.25; S, 13.92; found: C, 60.03; H, 4.38; N, 18.30; S, 13.96. IR (KBr), $v\left(\mathrm{~cm}^{-1}\right): 3205(\mathrm{NH}), 3114(\mathrm{CH}$ aromatic), 2978 (CH aliphatic), $1705(\mathrm{C}=\mathrm{O}), 1610(\mathrm{C}=\mathrm{N}), 1516(\mathrm{C}-\mathrm{N})$, $1337(\mathrm{C}=\mathrm{S}), 685(\mathrm{C}-\mathrm{S}) .{ }^{1} \mathrm{H}$ NMR (DMSO- $\left.d_{6}\right) \delta(\mathrm{ppm}): 4.00$ (s, 2H, $\left.\mathrm{CH}_{2}\right), 7.12-7.51$ (m, 15H, 15ArH), 9.38, 9.76, 10.47 (3brs, 3H, 3NH). ${ }^{13} \mathrm{C}$ NMR $\delta(\mathrm{ppm}): 34.55\left(-\mathrm{S}-\mathrm{CH}_{2}-\right)$, $125.23,125.79,126.45,127.77,127.92,128.09,128.75$, 130.07, 130.15 (15CH aromatic), 130.36, 133.78, 139.09 (3C aromatic), $151.75(\mathrm{C}-\mathrm{S}), 154.48(\mathrm{C}-3$ triazole $), 166.95(\mathrm{C}=\mathrm{O})$, $180.98(\mathrm{C}=\mathrm{S})$. MS $m / z(\%): 460\left(\mathrm{M}^{+}, 1\right), 383(1.2), 325(13)$, 294 (20), 252 (60), 194 (10), 180 (10), 149 (8), 135 (74), 131 (5), 104 (25), 91 (33), 77 (100).

4-(4-Bromophenyl)-1-\{[(4,5-diphenyl-4H-1,2,4-triazol-3yl)sulfanyl]acetyl\} thiosemicarbazide (4e) Yield: $88.3 \%$. Temperature of reaction: $110{ }^{\circ} \mathrm{C}$ for $16 \mathrm{~h}, \mathrm{mp}: 188-190{ }^{\circ} \mathrm{C}$ (dec.). Analysis for $\mathrm{C}_{23} \mathrm{H}_{19} \mathrm{BrN}_{6} \mathrm{OS}_{2}$ (539.47); calculated: C, 51.21; H, 3.55; N, 15.58; S, 11.88; Br, 14.81; found: C, 51.27; H, 3.54; N, 15.61; S, 11.92. IR (KBr), $v\left(\mathrm{~cm}^{-1}\right)$ : $3213(\mathrm{NH}), 3116$ (CH aromatic), 2972 (CH aliphatic), 1703 $(\mathrm{C}=\mathrm{O}), 1600(\mathrm{C}=\mathrm{N}), 1341(\mathrm{C}=\mathrm{S}), 690(\mathrm{C}-\mathrm{S}) .{ }^{1} \mathrm{H}$ NMR $\left(\right.$ DMSO- $\left.d_{6}\right) \delta(\mathrm{ppm}): 3.97\left(\mathrm{~s}, 2 \mathrm{H}, \mathrm{CH}_{2}\right), 7.29-7.55(\mathrm{~m}$, $14 \mathrm{H}, 14 \mathrm{ArH}), 9.79,9.82,10.46$ (3brs, 3H, 3NH).

4-(4-Chlorophenyl)-1-\{[(4,5-diphenyl-4H-1,2,4-triazol-3-yl) sulfanyl]acetyl\} thiosemicarbazide (4f) Yield: $97.8 \%$. Temperature of reaction: $100{ }^{\circ} \mathrm{C}$ for $16 \mathrm{~h}, \mathrm{mp}: 180-184{ }^{\circ} \mathrm{C}$ (dec.). Analysis for $\mathrm{C}_{23} \mathrm{H}_{19} \mathrm{ClN}_{6} \mathrm{OS}_{2}$ (495.02); calculated: $\mathrm{C}$,
55.80; H, 3.87; N, 16.98; S, 12.95; Cl, 9.16; found: C, 55.83; H, 3.88; N, 16.93; S, 12.90. IR (KBr), $v\left(\mathrm{~cm}^{-1}\right): 3202(\mathrm{NH})$, 3093 (CH aromatic), 2983 (CH aliphatic), 1705 (C=O), 1608 $(\mathrm{C}=\mathrm{N}), 1338(\mathrm{C}=\mathrm{S}), 688(\mathrm{C}-\mathrm{S}) .{ }^{1} \mathrm{H}$ NMR (DMSO- $\left.d_{6}\right) \delta$ (ppm): 3.98 (s, $\left.2 \mathrm{H}, \mathrm{CH}_{2}\right), 7.31-7.56(\mathrm{~m}, 14 \mathrm{H}, 14 \mathrm{ArH}), 9.81$, 9.88, 10.46 (3brs, $3 \mathrm{H}, 3 \mathrm{NH}) .{ }^{13} \mathrm{C}$ NMR $\delta$ (ppm): $34.39(-\mathrm{S}-$ $\left.\mathrm{CH}_{2}-\right), 121.95,125.63,128.40,128.56,128.76,129.37$, $129.54,130.08$ (14CH aromatic), 128.60, 130.19, 133.65, 137.92 (4C aromatic), $151.63(\mathrm{C}-\mathrm{S}), 154.30$ (C-3 triazole), $166.85(\mathrm{C}=\mathrm{O}), 180.84(\mathrm{C}=\mathrm{S})$.

4-(4-Methoxyphenyl)-1-\{[(4,5-diphenyl-4H-1,2,4-triazol-3yl)sulfanyl]acetyl\} thiosemicarbazide $(\mathbf{4 g}) \quad$ Yield: $95.2 \%$. Temperature of reaction: $60^{\circ} \mathrm{C}$ for $18 \mathrm{~h}$, mp: $172-174{ }^{\circ} \mathrm{C}(\mathrm{dec}$.). Analysis for $\mathrm{C}_{24} \mathrm{H}_{22} \mathrm{~N}_{6} \mathrm{O}_{2} \mathrm{~S}_{2}$ (490.60); calculated: C, 58.75; H, 4.52; N, 17.13; S, 13.07; found: C, 58.97; H, 4.51; N, 17.18; S, 13.10. IR (KBr), $v\left(\mathrm{~cm}^{-1}\right): 3198(\mathrm{NH}), 3102(\mathrm{CH}$ aromatic), 2988, 1452, 759 (CH aliphatic), $1710(\mathrm{C}=\mathrm{O}), 1605(\mathrm{C}=\mathrm{N}), 1519$ $(\mathrm{C}-\mathrm{N}), 1329(\mathrm{C}=\mathrm{S}), 693(\mathrm{C}-\mathrm{S}) .{ }^{1} \mathrm{H}$ NMR (DMSO- $\left.d_{6}\right) \delta(\mathrm{ppm})$ : 3.74 (s, $\left.3 \mathrm{H}, \mathrm{CH}_{3}\right), 3.99$ (s, $\left.2 \mathrm{H}, \mathrm{CH}_{2}\right), 6.90(\mathrm{~d}, J=6 \mathrm{~Hz}, 2 \mathrm{H}$, 2ArH), 7.32-7.56 (m, 10H, 10ArH), $7.57(\mathrm{~d}, J=6 \mathrm{~Hz}, 2 \mathrm{H}$, 2ArH), 9.61, 9.66, 10.40 (3brs, 3H, 3NH).

4-Benzyl-1-\{[(4,5-diphenyl-4H-1,2,4-triazol-3-yl)sulfanyl] acetyl\} thiosemicarbazide (4h) Yield: $95.0 \%$. Temperature of reaction: $50{ }^{\circ} \mathrm{C}$ for $12 \mathrm{~h}, \mathrm{mp}: 176-180{ }^{\circ} \mathrm{C}$ (dec.). Analysis for $\mathrm{C}_{24} \mathrm{H}_{22} \mathrm{~N}_{6} \mathrm{OS}_{2}$ (474.60); calculated: C, 60.74; $\mathrm{H}, 4.67$; N, 17.71; S, 13.51; found: C, 60.77; H, 4.66; N, 17.78; S, 13.55. IR (KBr), $v\left(\mathrm{~cm}^{-1}\right): 3209(\mathrm{NH}), 3087(\mathrm{CH}$ aromatic), 2971, 1439 (CH aliphatic), $1700(\mathrm{C}=\mathrm{O}), 1611$ $(\mathrm{C}=\mathrm{N}), 1520(\mathrm{C}-\mathrm{N}), 1351(\mathrm{C}=\mathrm{S}), 689(\mathrm{C}-\mathrm{S}) .{ }^{1} \mathrm{H}$ NMR $\left(\mathrm{DMSO}-d_{6}\right) \delta$ (ppm): $3.90\left(\mathrm{~s}, 2 \mathrm{H}, \mathrm{CH}_{2}\right), 4.84\left(\mathrm{~s}, 2 \mathrm{H}, \mathrm{CH}_{2}\right.$ ), 7.15-7.54 (m, 15H, 15ArH), 8.82, 9.54, 10.41 (3brs, 3H, $3 \mathrm{NH}) .{ }^{13} \mathrm{C}$ NMR $\delta(\mathrm{ppm}): 33.68\left(-\mathrm{S}-\mathrm{CH}_{2}-\right), 46.62\left(-\mathrm{CH}_{2}-\right.$ ), 126.47, 127.12, 127.46, 127.83, 128.16, 128.51, 128.83, $129.83,130.04$ (15CH aromatic), 133.71, 134.71, 139.34 (3C aromatic), 151.95 (C-S), 154.32 (C-3 triazole), 166.79 $(\mathrm{C}=\mathrm{O}), 182.09(\mathrm{C}=\mathrm{S})$.

4-(4-Methoxybenzyl)-1-\{[(4,5-diphenyl-4H-1,2,4-triazol-3$y$ l)sulfanyl]acetyl\} thiosemicarbazide (4i) Yield: $97.4 \%$. Temperature of reaction: $50{ }^{\circ} \mathrm{C}$ for $14 \mathrm{~h}, \mathrm{mp}: 176-178{ }^{\circ} \mathrm{C}$ (dec.). Analysis for $\mathrm{C}_{25} \mathrm{H}_{24} \mathrm{~N}_{6} \mathrm{O}_{2} \mathrm{~S}_{2}$ (504.63); calculated: $\mathrm{C}$, 59.50; H, 4.79; N, 16.65; S, 12.71; found: C, 59.61; H, 4.78; N, 16.68; S, 12.75. IR (KBr), $v\left(\mathrm{~cm}^{-1}\right): 3222(\mathrm{NH})$, $3102 \mathrm{CH}$ (aromatic), 2973, 1448, 767 (CH aliphatic), 1697 $(\mathrm{C}=\mathrm{O}), 1599(\mathrm{C}=\mathrm{N}), 1514(\mathrm{C}-\mathrm{N}), 1349(\mathrm{C}=\mathrm{S}), 680(\mathrm{C}-\mathrm{S})$. ${ }^{1} \mathrm{H}$ NMR (DMSO- $\left.d_{6}\right) \delta(\mathrm{ppm}): 3.76\left(\mathrm{~s}, 3 \mathrm{H}, \mathrm{CH}_{3}\right), 4.01$ (s, $\left.2 \mathrm{H}, \mathrm{CH}_{2}\right), 4.74\left(\mathrm{~s}, 2 \mathrm{H}, \mathrm{CH}_{2}\right), 6.86-7.64(\mathrm{~m}, 14 \mathrm{H}, 14 \mathrm{ArH})$, $8.33,9.55,10.44$ (3brs, $3 \mathrm{H}, 3 \mathrm{NH})$. 
4-Ethoxycarbonyl-1-\{[(4,5-diphenyl-4H-1,2,4-triazol-3-yl) sulfanyl]acetyl\} thiosemicarbazide (4j) Yield: $98.6 \%$. Temperature of reaction: $55^{\circ} \mathrm{C}$ for $14 \mathrm{~h}, \mathrm{mp}: 178-180{ }^{\circ} \mathrm{C}$ (dec.). Analysis for $\mathrm{C}_{20} \mathrm{H}_{20} \mathrm{~N}_{6} \mathrm{O}_{3} \mathrm{~S}_{2}$ (456.54); calculated: $\mathrm{C}$, 52.62; H, 4.41; N, 18.41; S, 14.05; found: C, 52.76; H, 4.42; N, 18.44; S, 14.01. IR (KBr), $v\left(\mathrm{~cm}^{-1}\right): 3219(\mathrm{NH})$, 3105 (CH aromatic), 2973, 1452, 765 (CH aliphatic), 1728 $(\mathrm{C}=\mathrm{O}$ acidic $), 1699(\mathrm{C}=\mathrm{O}), 1608(\mathrm{C}=\mathrm{N}), 1511(\mathrm{C}-\mathrm{N}), 1338$ $(\mathrm{C}=\mathrm{S}), 691(\mathrm{C}-\mathrm{S}) .{ }^{1} \mathrm{H}$ NMR (DMSO- $\left.d_{6}\right) \delta(\mathrm{ppm}): 1.22$ (t, $\left.J=5 \mathrm{~Hz}, 3 \mathrm{H}, \mathrm{CH}_{3}\right), 4.09$ (s, $\left.2 \mathrm{H}, \mathrm{CH}_{2}\right), 4.12-4.21$ (q, $\left.J=7.5 \mathrm{~Hz}, J=7.5 \mathrm{~Hz}, 2 \mathrm{H}, \mathrm{CH}_{2}\right), 7.28-7.56(\mathrm{~m}, 10 \mathrm{H}$, $10 \mathrm{ArH}), 11.07,11.38,11.51$ (3brs, $3 \mathrm{H}, 3 \mathrm{NH}$ ).

4-Ethoxycarbonylmethyl-1-\{[(4,5-diphenyl-4H-1,2, 4-triazol-3-yl)sulfanyl]acetyl\} thiosemicarbazide (4k) Yield: $91.9 \%$. Temperature of reaction: $50{ }^{\circ} \mathrm{C}$ for $14 \mathrm{~h}, \mathrm{mp}$ : $188-190{ }^{\circ} \mathrm{C}$ (dec.). Analysis for $\mathrm{C}_{21} \mathrm{H}_{22} \mathrm{~N}_{6} \mathrm{O}_{3} \mathrm{~S}_{2}$ (470.57); calculated: C, 53.60; H, 4.71; N, 17.86; S, 13.63; found: C, 53.46; H, 4.72; N, 17.90; S, 13.67. IR (KBr), $v\left(\mathrm{~cm}^{-1}\right)$ : 3211 (NH), 3096 (CH aromatic), 2975, 1464, 758 (CH aliphatic), $1737(\mathrm{C}=\mathrm{O}$ acidic $), 1703(\mathrm{C}=\mathrm{O}), 1611(\mathrm{C}=\mathrm{N}), 1511(\mathrm{C}-\mathrm{N})$, $1344(\mathrm{C}=\mathrm{S}), 686(\mathrm{C}-\mathrm{S}) .{ }^{1} \mathrm{H}$ NMR (DMSO- $\left.d_{6}\right) \delta(\mathrm{ppm}): 1.16$ $\left(\mathrm{t}, J=5 \mathrm{~Hz}, 3 \mathrm{H}, \mathrm{CH}_{3}\right), 3.96\left(\mathrm{~s}, 2 \mathrm{H}, \mathrm{CH}_{2}\right), 4.02-4.11(\mathrm{q}$, $\left.J=7.5 \mathrm{~Hz}, J=7.5 \mathrm{~Hz}, 2 \mathrm{H}, \mathrm{CH}_{2}\right), 4.30\left(\mathrm{~s}, 2 \mathrm{H}, \mathrm{CH}_{2}\right)$, 7.33-7.58 (m, 10H, 10ArH), 8.78, 9.69, 10.47 (3brs, 3H, $3 \mathrm{NH})$

4-Benzoyl-1-\{[(4,5-diphenyl-4H-1,2,4-triazol-3-yl)sulfanyl]acetyl\} thiosemicarbazide (4l) Yield: $96.8 \%$. Temperature of reaction: $50{ }^{\circ} \mathrm{C}$ for $20 \mathrm{~h}$, mp: $180-182{ }^{\circ} \mathrm{C}$ (dec.). Analysis for $\mathrm{C}_{24} \mathrm{H}_{20} \mathrm{~N}_{6} \mathrm{O}_{2} \mathrm{~S}_{2}$ (488.58); calculated: C, 59.00; H, 4.13; N, 17.20; S, 13.12; found: C, 58.95; H, 4.12; N, 17.26; S, 13.08. IR (KBr), $v\left(\mathrm{~cm}^{-1}\right): 3176(\mathrm{NH}), 3088(\mathrm{CH}$ aromatic), 2979, 1449 ( $\mathrm{CH}$ aliphatic), 1746 ( $\mathrm{C}=\mathrm{O}$ acidic), $1703(\mathrm{C}=\mathrm{O})$, $1608(\mathrm{C}=\mathrm{N}), 1509(\mathrm{C}-\mathrm{N}), 1311(\mathrm{C}=\mathrm{S}), 681(\mathrm{C}-\mathrm{S}) .{ }^{1} \mathrm{H}$ NMR (DMSO- $\left.d_{6}\right) \delta$ (ppm): 4.15 (s, 2H, $\mathrm{CH}_{2}$ ), 7.35-7.96 (m, 15H, $15 \mathrm{ArH}), 11.33,11.77,12.87$ (3brs, 3H, 3NH).

Derivatives of 4,5-disubstituted-1,2,4-triazole-3(2H)thione $(\mathbf{5} \mathbf{a}-\mathbf{i})$

\section{General procedure}

A mixture of thiosemicarbazide $\mathbf{4 a}-\mathbf{i}(10 \mathrm{mmol})$ and $20-40 \mathrm{~mL}$ of $2 \%$ aqueous solution of sodium hydroxide was refluxed for $2 \mathrm{~h}$. Then, the solution was neutralized with diluted hydrochloric acid and the formed precipitate was filtered and crystallized from ethanol $\mathbf{5 c}, \mathbf{d}, \mathbf{h}, \mathbf{i}$, butanol 5b, e, f, or methanol 5a, g.

4-Ethyl-5-\{[(4,5-diphenyl-4H-1,2,4-triazol-3-yl)sulfanyl] methyl $\}-4 H-1,2,4-t r i a z o l e-3(2 H)$-thione $\quad(5 a)$ Yield: $87.6 \%$, mp: $214-216{ }^{\circ} \mathrm{C}$ (dec.). Analysis for $\mathrm{C}_{19} \mathrm{H}_{18} \mathrm{~N}_{6} \mathrm{~S}_{2}$ (394.52); calculated: C, 57.84; H, 4.60; N, 21.30; S, 16.25; found: C, 57.67; H, 4.59; N, 21.33; S, 16.21. IR (KBr), v $\left(\mathrm{cm}^{-1}\right)$ : $3135(\mathrm{NH}), 3085$ (CH aromatic), 2958, 1422, 758 (CH aliphatic), $1600(\mathrm{C}=\mathrm{N}), 1502(\mathrm{C}-\mathrm{N}), 1350(\mathrm{C}=\mathrm{S}), 692$ $(\mathrm{C}-\mathrm{S}) .{ }^{1} \mathrm{H}$ NMR (DMSO- $\left.d_{6}\right) \delta$ (ppm): $1.22(\mathrm{t}, J=5 \mathrm{~Hz}$, $\left.3 \mathrm{H}, \mathrm{CH}_{3}\right), 3.91-3.97$ (q, $J=5 \mathrm{~Hz}, J=5 \mathrm{~Hz}, 2 \mathrm{H}, \mathrm{CH}_{2}$ ), $4.39\left(\mathrm{~s}, 2 \mathrm{H}, \mathrm{CH}_{2}\right), 7.27-7.54(\mathrm{~m}, 10 \mathrm{H}, 10 \mathrm{ArH}), 13.62(\mathrm{~s}$, $1 \mathrm{H}, \mathrm{NH})$. MS m/z (\%): $394\left(\mathrm{M}^{+}, 0.2\right), 365(0.1), 339$ (0.12), 264 (0.1), 253 (64), 252 (68), 194 (21), 149 (33), 128 (16), 118 (37), 104 (10), 91 (58), 77 (100).

4-Allyl-5-\{[(4,5-diphenyl-4H-1,2,4-triazol-3-yl)sulfanyl]methyl\}-4H-1,2,4-triazole-3(2H)-thione $(\mathbf{5 b})$ Yield: 90.5\%, mp: $207-208{ }^{\circ} \mathrm{C}$ (dec.). Analysis for $\mathrm{C}_{20} \mathrm{H}_{18} \mathrm{~N}_{6} \mathrm{~S}_{2}$ (406.53); calculated: C, 59.10; H, 4.46; N, 20.67; S, 15.77; found: C, 58.96; H, 4.45; N, 20.64; S, 15.74. IR (KBr), $v\left(\mathrm{~cm}^{-1}\right): 3185$ (NH), 3091 (CH aromatic), 2989, 1450, 756 (CH aliphatic), 1604 $(\mathrm{C}=\mathrm{N}), 1510(\mathrm{C}-\mathrm{N}), 1343(\mathrm{C}=\mathrm{S}), 684(\mathrm{C}-\mathrm{S}) .{ }^{1} \mathrm{H}$ NMR (DMSO$\left.d_{6}\right) \delta(\mathrm{ppm}): 4.44\left(\mathrm{~s}, 2 \mathrm{H}, \mathrm{CH}_{2}\right), 4.69-4.71\left(\mathrm{~d}, J=5 \mathrm{~Hz}, 2 \mathrm{H}, \mathrm{CH}_{2}\right)$, $5.24-5.41\left(\mathrm{dd}, J=5 \mathrm{~Hz}, J=5 \mathrm{~Hz}, 2 \mathrm{H},=\mathrm{CH}_{2}\right), 5.82-5.93(\mathrm{~m}$, $1 \mathrm{H}, \mathrm{CH}), 7.37-7.62$ (m, 10H, 10ArH), 13.81 (brs, 1H, NH).

4-Cyclohexyl-5-\{[(4,5-diphenyl-4H-1,2,4-triazol-3-yl)sulfa -nyl]methyl\}-4H-1,2,4-triazole-3(2H)-thione (5c) Yield: 62.4 \%, mp: $186-188{ }^{\circ} \mathrm{C}$ (dec.). Analysis for $\mathrm{C}_{23} \mathrm{H}_{24} \mathrm{~N}_{6} \mathrm{~S}_{2}$ (448.61); calculated: C, 61.58; H, 5.39; N, 18.73; S, 14.29; found: $\mathrm{C}, 61.37 ; \mathrm{H}, 5.38 ; \mathrm{N}, 18.68 ; \mathrm{S}, 14.32$. IR $(\mathrm{KBr}), v\left(\mathrm{~cm}^{-1}\right)$ : $3175(\mathrm{NH}), 3088(\mathrm{CH}$ aromatic), 2963, 1449, $759(\mathrm{CH}$ aliphatic), $1611(\mathrm{C}=\mathrm{N}), 1505(\mathrm{C}-\mathrm{N}), 1339(\mathrm{C}=\mathrm{S}), 684(\mathrm{C}-\mathrm{S}) .{ }^{1} \mathrm{H}$ NMR (DMSO- $\left.d_{6}\right) \delta$ (ppm): $1.05-1.73\left(\mathrm{~m}, 10 \mathrm{H}, 5 \mathrm{CH}_{2}\right.$ cyclohexane), $4.04\left(\mathrm{~s}, 2 \mathrm{H}, \mathrm{CH}_{2}\right), 4.45(\mathrm{~m}, 1 \mathrm{H}, \mathrm{CH}$ cyclohexane), 7.29-7.56 (m, 10H, 10ArH), 14.13 (brs, 1H, NH).

4-Phenyl-5-\{[(4,5-diphenyl-4H-1,2,4-triazol-3-yl)sulfanyl] methyl)-4H-1,2,4-triazole-3(2H)-thione (5d) Yield: $76.9 \%$, mp: $209-210{ }^{\circ} \mathrm{C}$ (dec.). Analysis for $\mathrm{C}_{23} \mathrm{H}_{18} \mathrm{~N}_{6} \mathrm{~S}_{2}$ (442.56); calculated: C, 62.42; H, 4.10; N, 18.99; S, 14.49; found: C, 62.28; H, 4.09; N, 18.93; S, 14.51. IR (KBr), v $\left(\mathrm{cm}^{-1}\right): 3175(\mathrm{NH}), 3090(\mathrm{CH}$ aromatic), $2972(\mathrm{CH}$ aliphatic), $1598(\mathrm{C}=\mathrm{N}), 1505(\mathrm{C}-\mathrm{N}), 1326(\mathrm{C}=\mathrm{S}), 684(\mathrm{C}-\mathrm{S})$. ${ }^{1} \mathrm{H} \mathrm{NMR}$ (DMSO- $\left.d_{6}\right) \delta$ (ppm): 4.14 (s, $2 \mathrm{H}, \mathrm{CH}_{2}$ ), 7.12-7.59 (m, 15H, 15ArH), 13.86 (brs, $1 \mathrm{H}, \mathrm{NH}) .{ }^{13} \mathrm{C}$ NMR $\delta$ (ppm): $26.22\left(-\mathrm{S}-\mathrm{CH}_{2}-\right), 125.61,128.44,128.55,128.63,128.74$, $129.23,129.41,129.58,130.11$ (15CH aromatic), 138.23, 146.83, 148.15 (3C aromatic), 150.65 (C-3' triazole), 153.33 (C-S), 166.98 (C-3 triazole), 167.42 (C=S). MS $m / z(\%): 442$ $\left(\mathrm{M}^{+}, 2\right), 306$ (1), 294 (1), 252 (98), 194 (23), 149 (18), 127 (14), 118 (44), 104 (8), 91 (27), 77 (100).

4-(4-Bromophenyl)-5-\{[(4,5-diphenyl-4H-1,2,4-triazol-3yl)sulfanyl]methyl\}-4H-1,2,4-triazole-3(2H)-thione (5e) Yield: $97.2 \%$, mp: $210-212{ }^{\circ} \mathrm{C}$ (dec.). Analysis for $\mathrm{C}_{23} \mathrm{H}_{17} \mathrm{BrN}_{6} \mathrm{~S}_{2}$ (521.45); calculated: $\mathrm{C}, 52.98 ; \mathrm{H}, 3.29 ; \mathrm{N}$, 
16.12; S, 12.30; Br, 15.32; found: C, 52.93; H, 3.28; N, 16.15; S, 12.32. IR (KBr), $v\left(\mathrm{~cm}^{-1}\right): 3178(\mathrm{NH}), 3102(\mathrm{CH}$ aromatic), 2965, 1448 ( $\mathrm{CH}$ aliphatic), $1609(\mathrm{C}=\mathrm{N}), 1504$ $(\mathrm{C}-\mathrm{N}), 1367(\mathrm{C}=\mathrm{S}), 688(\mathrm{C}-\mathrm{S}) .{ }^{1} \mathrm{H}$ NMR (DMSO-d $)_{6} \delta$ (ppm): 4.17 (s, 2H, $\left.\mathrm{CH}_{2}\right), 7.14-7.46(\mathrm{~m}, 14 \mathrm{H}, 14 \mathrm{ArH})$, 13.89 (brs, 1H, NH).

4-(4-Chlorophenyl)-5-\{[(4,5-diphenyl-4H-1,2,4-triazol-3yl)sulfanyl]methyl\}-4H-1,2,4-triazole-3(2H)-thione (5f) Yield: $96.0 \%$, mp: $118-120{ }^{\circ} \mathrm{C}$ (dec.). Analysis for $\mathrm{C}_{23} \mathrm{H}_{17} \mathrm{ClN}_{6} \mathrm{~S}_{2}$ (477.00); calculated: C, 57.91; H, 3.59; N, 17.62; S, 13.44; Cl, 7.43; found: C, 57.85; H, 3.58; N, 17.65; S, 13.41. IR (KBr), $v\left(\mathrm{~cm}^{-1}\right): 3143(\mathrm{NH}), 3088(\mathrm{CH}$ aromatic), 2985, 1459 ( $\mathrm{CH}$ aliphatic), $1601(\mathrm{C}=\mathrm{N}), 1500$ $(\mathrm{C}-\mathrm{N}), 1361(\mathrm{C}=\mathrm{S}), 690(\mathrm{C}-\mathrm{S}) .{ }^{1} \mathrm{H}$ NMR (DMSO-d $) \delta$ (ppm): 4.17 (s, 2H, $\mathrm{CH}_{2}$ ), 7.22-7.58 (m, 14H, 14ArH), 13.89 (brs, 1H, NH).

4-(4-Methoxyphenyl)-5-\{[(4,5-diphenyl-4H-1,2,4-triazol-3yl)sulfanyl]methyl\}-4H-1,2,4-triazole-3(2H)-thione (5g) Yield: $98.3 \%$, mp: 206-208 ${ }^{\circ} \mathrm{C}$ (dec.). Analysis for $\mathrm{C}_{24} \mathrm{H}_{20} \mathrm{~N}_{6} \mathrm{OS}_{2}$ (472.58); calculated: C, 60.99; H, 4.26; N, 17.78; S, 13.57; found: C, 61.16; H, 4.25; N, 17.71; S, 13.61. IR (KBr), $v\left(\mathrm{~cm}^{-1}\right): 3164(\mathrm{NH}), 3094(\mathrm{CH}$ aromatic), 2969, 1441 (CH aliphatic), $1612(\mathrm{C}=\mathrm{N}), 1506(\mathrm{C}-\mathrm{N}), 1319$ $(\mathrm{C}=\mathrm{S}), 691(\mathrm{C}-\mathrm{S}) .{ }^{1} \mathrm{H}$ NMR (DMSO- $\left.d_{6}\right) \delta(\mathrm{ppm}): 3.80(\mathrm{~s}$, $\left.3 \mathrm{H}, \mathrm{CH}_{3}\right), 4.14$ (s, 2H, $\left.\mathrm{CH}_{2}\right), 7.03-7.59$ (m, 14H, 14ArH), 13.82 (brs, $1 \mathrm{H}, \mathrm{NH}) .{ }^{13} \mathrm{C} \mathrm{NMR} \delta$ (ppm): $27.55\left(-\mathrm{S}-\mathrm{CH}_{2}-\right)$, $55.56\left(\mathrm{CH}_{3}\right), 114.56,125.67,127.62,128.33,128.67$, 129.32, 129.55, 130.11 (14CH aromatic), 133.71, 134.62, 139.61, 159.81 (4C aromatic), 150.51 (C-3' triazole), $154.73(\mathrm{C}-\mathrm{S}), 168.42$ (C-3 triazole), $168.61(\mathrm{C}=\mathrm{S})$. MS m/ z (\%): $472\left(\mathrm{M}^{+}, 0.07\right), 457$ (0.03), 440 (0.02), 339 (0.21), 306 (0.05), 294 (0.11), 268 (11), 252 (59), 209 (7), 194 (15), 149 (12), 135 (86), 118 (13), 104 (10), 91 (27), 77 (100).

\section{4-Benzyl-5-\{[(4,5-diphenyl-4H-1,2,4-triazol-3-yl)sulfanyl $]$} methyl\}-4H-1,2,4-triazole-3(2H)-thione (5h) Yield: $79.0 \%$, mp: $136-140{ }^{\circ} \mathrm{C}$ (dec.). Analysis for $\mathrm{C}_{24} \mathrm{H}_{20} \mathrm{~N}_{6} \mathrm{~S}_{2}$ (456.58); calculated: C, 63.13; H, 4.41; N, 18.41; S, 14.04; found: C, 63.26; H, 4.42; N, 18.35; S, 14.08. IR (KBr), $v$ $\left(\mathrm{cm}^{-1}\right): 3155(\mathrm{NH}), 3091(\mathrm{CH}$ aromatic) 2961, 1453, 762 (CH aliphatic), $1609(\mathrm{C}=\mathrm{N}), 1508(\mathrm{C}-\mathrm{N}), 1342(\mathrm{C}=\mathrm{S}), 677$ $(\mathrm{C}-\mathrm{S}) .{ }^{1} \mathrm{H}$ NMR (DMSO-d $)_{6} \delta$ (ppm): 4.29 (s, 2H, $\mathrm{CH}_{2}$ ), 5.24 (s, 2H, $\mathrm{CH}_{2}$ ), 7.22-7.53 (m, 15H, 15ArH), 13.86 (brs, $1 \mathrm{H}, \mathrm{NH})$.

4-(4-Methoxybenzyl)-5-\{[(4,5-diphenyl-4H-1,2,4-triazol-3yl)sulfanyl]methyl\}-4H-1,2,4-triazole-3(2H)-thione Yield: $98.5 \%$, mp: $118-120{ }^{\circ} \mathrm{C}$ (dec.). Analysis for $\mathrm{C}_{25} \mathrm{H}_{22} \mathrm{~N}_{6} \mathrm{OS}_{2}$ (486.61); calculated: $\mathrm{C}, 61.70 ; \mathrm{H}, 4.56 ; \mathrm{N}$, 17.27; S, 13.18; found: C, 61.61; H, 4.55; N, 17.25; S,
13.14. IR $(\mathrm{KBr}), v\left(\mathrm{~cm}^{-1}\right): 3174(\mathrm{NH}), 3071(\mathrm{CH}$ aromatic), 2982, 1453, $764(\mathrm{CH}$ aliphatic), $1612(\mathrm{C}=\mathrm{N}), 1510$ $(\mathrm{C}-\mathrm{N}), 1358(\mathrm{C}=\mathrm{S}), 673(\mathrm{C}-\mathrm{S}) .{ }^{1} \mathrm{H}$ NMR $\left(\mathrm{DMSO}-d_{6}\right) \delta$ (ppm): $3.71\left(\mathrm{~s}, 3 \mathrm{H}, \mathrm{CH}_{3}\right), 4.33\left(\mathrm{~s}, 2 \mathrm{H}, \mathrm{CH}_{2}\right), 5.20(\mathrm{~s}, 2 \mathrm{H}$, $\mathrm{CH}_{2}$ ), 6.83-7.52 (m, 14H, 14ArH), 13.82 (brs, 1H, NH).

Derivatives of 2,5-disubstituted-1,3,4-thiadiazole (6a-i)

Method A (for compounds $\boldsymbol{6} \boldsymbol{a}-\boldsymbol{i})$

$10 \mathrm{mmol}$ of 4-substituted-1-\{[(4,5-diphenyl-4H-1,2,4-triazol-3-yl)sulfanyl]acetyl $\}$ thiosemicarbazide $\mathbf{4 a - i}$ was dissolved in 10-20 mL diluted sulfuric acid and stirred in a closed bulb for $1 \mathrm{~h}$. Subsequently, the solution was poured out on crushed ice $(50 \mathrm{~g})$ and stirred until the ice was completely dissolved. Later, the solution was neutralized with ammonium hydroxide. The precipitate that formed was filtered, dried, and crystallized from ethanol $\mathbf{6 a}, \mathbf{c}, \mathbf{d}$, $\mathbf{g - i}$ or butanol $\mathbf{6 b}, \mathbf{e}, \mathbf{f}$.

\section{Method B (for compounds $\mathbf{6} \boldsymbol{a}, \boldsymbol{d})$}

$20 \mathrm{~mL}$ of $10 \%$ ethanolic solution of hydrochloric acid was added to thiosemicarbazide $\mathbf{4 a}, \mathbf{d}$ and the reaction mixture was heated under reflux for $1 \mathrm{~h}$. Subsequently, the solution was left at room temperature for $24 \mathrm{~h}$. The precipitate formed was separated by filtration, dried, and crystallized from ethanol.

\section{Method $C$ (for compounds $\boldsymbol{\sigma e}, \boldsymbol{f})$}

A mixture of $10 \mathrm{mmol}$ of thiosemicarbazide $4 \mathbf{e}, \mathbf{f}$ in $10 \mathrm{~mL}$ of anhydrous acetic acid was refluxed for $1 \mathrm{~h}$. Subsequently, the solution was left at room temperature for $12 \mathrm{~h}$. The precipitate that formed was separated by filtration, dried, and crystallized from butanol.

5-Aminoethyl-2-\{[(4,5-diphenyl-4H-1,2,4-triazol-3-yl)sulfanyl]methyl\}-1,3,4-thiadiazole (6a) Yield: $81.3 \%$, mp: $168-170{ }^{\circ} \mathrm{C}$ (dec.). Analysis for $\mathrm{C}_{19} \mathrm{H}_{18} \mathrm{~N}_{6} \mathrm{~S}_{2}$ (394.52); calculated: C, 57.84; H, 4.60; N, 21.30; S, 16.25; found: C, 57.69; H, 4.58; N, 21.26; S, 16.21. IR (KBr), $v\left(\mathrm{~cm}^{-1}\right)$ : $3244(\mathrm{NH}), 3071$ (CH aromatic), 2944, 1458, $733(\mathrm{CH}$ aliphatic), $1602(\mathrm{C}=\mathrm{N}), 1506(\mathrm{C}-\mathrm{N}), 671(\mathrm{C}-\mathrm{S}) .{ }^{1} \mathrm{H}$ NMR $\left(\mathrm{DMSO}-d_{6}\right) \delta$ (ppm): $1.13\left(\mathrm{t}, J=7.5 \mathrm{~Hz}, 3 \mathrm{H}, \mathrm{CH}_{3}\right)$, $3.21-3.27$ (q, $\left.J=5 \mathrm{~Hz}, J=5 \mathrm{~Hz}, 2 \mathrm{H}, \mathrm{CH}_{2}\right), 4.57$ (s, 2H, $\mathrm{CH}_{2}$ ), 7.17-7.70 (m, 10H, 10ArH), 9.35 (brs, 1H, NH).

5-Aminoallyl-2-\{[(4,5-diphenyl-4H-1,2,4-triazol-3-yl)sulfanyl]methyl\}-1,3,4-thiadiazole (6b) Yield: $68.9 \%$, mp: $208-210{ }^{\circ} \mathrm{C}$ (dec.). Analysis for $\mathrm{C}_{20} \mathrm{H}_{18} \mathrm{~N}_{6} \mathrm{~S}_{2}$ (406.53); calculated: C, 59.09; H, 4.46; N, 20.67; S, 15.77; found: C, 59.22; H, 4.45; N, 20.65; S, 15.73. IR (KBr), $v\left(\mathrm{~cm}^{-1}\right)$ : 
3256 (NH), 3083 (CH aromatic), 2955, 1489, $741(\mathrm{CH}$ aliphatic), $1610(\mathrm{C}=\mathrm{N}), 1503(\mathrm{C}-\mathrm{N}), 679(\mathrm{C}-\mathrm{S}) .{ }^{1} \mathrm{H}$ NMR $\left(\mathrm{DMSO}-d_{6}\right) \delta(\mathrm{ppm}): 3.87\left(\mathrm{~s}, 2 \mathrm{H}, \mathrm{CH}_{2}\right), 4.12(\mathrm{~d}, J=5 \mathrm{~Hz}$, $\left.2 \mathrm{H}, \mathrm{CH}_{2}\right), 5.02-5.13\left(\mathrm{dd}, J=5 \mathrm{~Hz}, J=5 \mathrm{~Hz}, 2 \mathrm{H},=\mathrm{CH}_{2}\right.$ ), 5.79-5.88 (m, 1H, CH), 7.40-8.56 (m, 10H, 10ArH), 10.13 (brs, $1 \mathrm{H}, \mathrm{NH}$ ).

5-Aminocyclohexyl-2-\{[(4,5-diphenyl-4H-1,2,4-triazol-3-yl) sulfanyl]methyl\}-1,3,4-thiadiazole (6c) Yield: $75.6 \%, \mathrm{mp}$ : $172-174{ }^{\circ} \mathrm{C}$ (dec.). Analysis for $\mathrm{C}_{23} \mathrm{H}_{24} \mathrm{~N}_{6} \mathrm{~S}_{2}$ (448.61); calculated: C, 61.58; H, 5.39; N, 18.73; S, 14.30; found: C, 61.61; H, 5.37; N, 18.76; S, 14.27. IR (KBr), $v\left(\mathrm{~cm}^{-1}\right): 3190$ (NH), 3093 (CH aromatic), 2972, 1467, 749 ( $\mathrm{CH}$ aliphatic), $1620(\mathrm{C}=\mathrm{N}), 681(\mathrm{C}-\mathrm{S}) .{ }^{1} \mathrm{H}$ NMR (DMSO- $\left.d_{6}\right) \delta(\mathrm{ppm})$ : 1.1-1.65 (m, 10H, 5CH $\mathrm{CH}_{2}$ cyclohexane), $3.03(\mathrm{~m}, 1 \mathrm{H}, \mathrm{CH}$ cyclohexane), $4.22\left(\mathrm{~s}, 2 \mathrm{H}, \mathrm{CH}_{2}\right), 7.33-8.06(\mathrm{~m}, 10 \mathrm{H}$, 10ArH), 10.16 (brs, 1H, NH).

5-Aminophenyl-2-\{[(4,5-diphenyl-4H-1,2,4-triazol-3-yl) sulfanyl]methyl\}-1,3,4-thiadiazole (6d) Yield: $50.9 \%$, mp: $192-198{ }^{\circ} \mathrm{C}$ (dec.). Analysis for $\mathrm{C}_{23} \mathrm{H}_{18} \mathrm{~N}_{6} \mathrm{~S}_{2}$ (442.60); calculated: C, 62.42; H, 4.10; N, 19.00; S, 14.49; found: C, 62.36; H, 4.09; N, 18.97; S, 14.53. IR (KBr), $v\left(\mathrm{~cm}^{-1}\right)$ : $3199(\mathrm{NH}), 3011$ (CH aromatic), 2968 (CH aliphatic), 1610 $(\mathrm{C}=\mathrm{N}), 1504(\mathrm{C}-\mathrm{N}), 683(\mathrm{C}-\mathrm{S}) .{ }^{1} \mathrm{H}$ NMR $\left(\mathrm{DMSO}-d_{6}\right) \delta$ (ppm): 4.02 (s, 2H, $\mathrm{CH}_{2}$ ), 6.98-7.54 (m, 15H, 15ArH), 10.42 (brs, $1 \mathrm{H}, \mathrm{NH}$ ).

[5-Amino-(4-bromophenyl)]-2-\{[(4,5-diphenyl-4H-1,2, 4-triazol-3-yl)sulfanyl]methyl\}-1,3,4-thiadiazole (6e) Yield: $89.4 \%$, mp: $203-205{ }^{\circ} \mathrm{C}$ (dec.). Analysis for $\mathrm{C}_{23} \mathrm{H}_{17} \mathrm{BrN}_{6} \mathrm{~S}_{2}$ (521.45); calculated: C, 52.98; H, 3.29; N, 16.12; S, 12.30; Br, 15.32; found: C, 52.73; H, 3.27; N, 16.15; S, 12.27. IR (KBr), $v\left(\mathrm{~cm}^{-1}\right): 3167(\mathrm{NH}), 3110(\mathrm{CH}$ aromatic), 2954, 1441 (CH aliphatic), $1602(\mathrm{C}=\mathrm{N}), 680(\mathrm{C}-$ $\mathrm{S}) .{ }^{1} \mathrm{H}$ NMR (DMSO- $\left.d_{6}\right) \delta(\mathrm{ppm}): 4.22\left(\mathrm{~s}, 2 \mathrm{H}, \mathrm{CH}_{2}\right)$, 6.89-7.65 (m, 14H, 14ArH), 10.23 (brs, 1H, NH).

[5-Amino-(4-chlorophenyl)]-2-\{[(4,5-diphenyl-4H-1,2, 4-triazol-3-yl)sulfanyl]methyl\}-1,3,4-thiadiazole

(6f)

Yield: $94.7 \%$, mp: $215-218{ }^{\circ} \mathrm{C}$ (dec.). Analysis for $\mathrm{C}_{23} \mathrm{H}_{17} \mathrm{ClN}_{6} \mathrm{~S}_{2}$ (477.00); calculated: C, 57.91; H, 3.59; N, 17.62; S, 13.44; Cl, 7.43; found: C, 57.71; H, 3.60; N, 17.58; $\mathrm{S}, 13.39$. IR $(\mathrm{KBr}), v\left(\mathrm{~cm}^{-1}\right)$ : $3245(\mathrm{NH}), 3065(\mathrm{CH}$ aromatic), 2977 (CH aliphatic), $1611(\mathrm{C}=\mathrm{N}), 1506(\mathrm{C}-\mathrm{N}), 695$ (C-S). ${ }^{1} \mathrm{H}$ NMR (DMSO-d $\left.{ }_{6}\right) \delta$ (ppm): 3.89 (s, 2H, $\mathrm{CH}_{2}$ ), 7.39-7.64 (m, 14H, 14ArH), 10.36 (brs, 1H, NH).

[5-Amino-(4-methoxyphenyl)]-2-\{[(4,5-diphenyl-4H-1,2, 4-triazol-3-yl)sulfanyl]methyl\}-1,3,4-thiadiazole (6g) Yield: $53.6 \%$, mp: $152-154{ }^{\circ} \mathrm{C}$ (dec.). Analysis for $\mathrm{C}_{24} \mathrm{H}_{20} \mathrm{~N}_{6} \mathrm{OS}_{2}$ (472.58); calculated: C, 60.99; H, 4.26; N,
17.78; S, 13.57; found: C, 60.89; H, 4.26; N, 17.75; S, 14.55 . IR $(\mathrm{KBr}), v\left(\mathrm{~cm}^{-1}\right): 3211(\mathrm{NH}), 3038(\mathrm{CH}$ aromatic), 2982, 1451 (CH aliphatic), $1600(\mathrm{C}=\mathrm{N}), 1502(\mathrm{C}-\mathrm{N}), 692(\mathrm{C}-\mathrm{S}) .{ }^{1} \mathrm{H}$ NMR (DMSO- $\left.d_{6}\right) \delta(\mathrm{ppm}): 3.71\left(\mathrm{~s}, 3 \mathrm{H}, \mathrm{CH}_{3}\right), 4.65(\mathrm{~s}, 2 \mathrm{H}$, $\mathrm{CH}_{2}$ ), 6.89-7.78 (m, 14H, 14ArH), 10.07 (brs, 1H, NH).

5-Aminobenzyl-2-\{[(4,5-diphenyl-4H-1,2,4-triazol-3-yl) sulfanyl]methyl\}-1,3,4-thiadiazole (6h) Yield: $70.2 \%$, mp: $146-148{ }^{\circ} \mathrm{C}$ (dec.). Analysis for $\mathrm{C}_{24} \mathrm{H}_{20} \mathrm{~N}_{6} \mathrm{~S}_{2}$ (456.58); calculated: C, 63.13; H, 4.41; N, 18.41; S, 14.04; found: C, 63.05; H, 4.39; N, 18.36; S, 14.09. IR (KBr), $v\left(\mathrm{~cm}^{-1}\right)$ : $3272(\mathrm{NH}), 3042$ (CH aromatic), 2934, 1458 (CH aliphatic), $1601(\mathrm{C}=\mathrm{N}), 1512(\mathrm{C}-\mathrm{N}), 686(\mathrm{C}-\mathrm{S}) .{ }^{1} \mathrm{H}$ NMR $\left(\mathrm{DMSO}-d_{6}\right) \delta$ (ppm): 4.11 (s, 2H, $\left.\mathrm{CH}_{2}\right), 4.73\left(\mathrm{~s}, 2 \mathrm{H}, \mathrm{CH}_{2}\right)$, 7.34-7.62 (m, 15H, 15ArH), 10.47 (brs, 1H, NH).

[5-Amino-(4-methoxybenzyl)]-2-\{[(4,5-diphenyl-4H-1,2, 4-triazol-3-yl)sulfanyl]methyl\}-1,3,4-thiadiazole

Yield: $71.4 \%$, mp: $218-220{ }^{\circ} \mathrm{C}$ (dec.). Analysis for $\mathrm{C}_{25} \mathrm{H}_{22} \mathrm{~N}_{6} \mathrm{OS}_{2}$ (486.61); calculated: C, 61.70; H, 4.56; N, 17.27; S, 13.18; found: C, 61.77; H, 4.55; N, 17.23; S, 13.22. IR (KBr), $v\left(\mathrm{~cm}^{-1}\right): 3268(\mathrm{NH}), 3095(\mathrm{CH}$ aromatic), 2955, 1420, 765 (CH aliphatic), $1598(\mathrm{C}=\mathrm{N}), 1508(\mathrm{C}-\mathrm{N}), 690(\mathrm{C}-\mathrm{S}) .{ }^{1} \mathrm{H}$ NMR $\left(\mathrm{DMSO}-d_{6}\right) \delta$ (ppm): $3.68\left(\mathrm{~s}, 3 \mathrm{H}, \mathrm{CH}_{3}\right), 3.98\left(\mathrm{~s}, 2 \mathrm{H}, \mathrm{CH}_{2}\right), 4.44$ (s, 2H, $\mathrm{CH}_{2}$ ), 6.86-7.64 (m, 14H, 14ArH), 10.44 (brs, 1H, NH).

Derivatives of $N, N$-disubstituted acetamide $(\mathbf{7} \mathbf{a}-\mathbf{i})$

General method (for compounds $7 \boldsymbol{a}-\boldsymbol{i}$ )

A mixture of $10 \mathrm{mmol}$ of appropriate 2,5-disubstituted-1, 3,4-thiadiazole $\mathbf{6 a}-\mathbf{i}$ in $5 \mathrm{~mL}$ of acetic anhydride was heated under reflux for $2 \mathrm{~h}$. Distilled water was added to the reaction mixture and it was allowed to cool. The resulting precipitate was filtered and washed with distilled water. The residue was purified by recrystallization from ethanol.

$N-(5-\{[(4,5-d i p h e n y l-4 H-1,2,4-t r i a z o l-3-y l)$ sulfanyl $] m e t h y l\}-$ 1,3,4-thiadiazol-2-yl)-N-ethylacetamide (7a) Yield: $75.6 \%$, mp: $182-184{ }^{\circ} \mathrm{C}$ (dec.). Analysis for $\mathrm{C}_{21} \mathrm{H}_{20} \mathrm{~N}_{6} \mathrm{OS}_{2}$ (436.55); calculated: C, 57.78; H, 4.62; N, 19.25; S, 14.69; found: C, 57.81; H, 4.61; N, 19.28; S, 14.69. IR ( $\mathrm{KBr})$, $v\left(\mathrm{~cm}^{-1}\right): 3091(\mathrm{CH}$ aromatic $), 2922,1467,742(\mathrm{CH}$ aliphatic), $1701(\mathrm{C}=\mathrm{O}), 1610(\mathrm{C}=\mathrm{N}), 1512(\mathrm{C}-\mathrm{N}), 692(\mathrm{C}-\mathrm{S})$. ${ }^{1} \mathrm{H}$ NMR (DMSO- $\left.d_{6}\right) \delta(\mathrm{ppm}): 1.31(\mathrm{t}, J=7.5 \mathrm{~Hz}, 3 \mathrm{H}$, $\mathrm{CH}_{3}$ ), 2.15 (s, 3H, $\mathrm{CH}_{3}$ ), 3.65-3.70 (q, $J=5 \mathrm{~Hz}, J=5 \mathrm{~Hz}$, $\left.2 \mathrm{H}, \mathrm{CH}_{2}\right), 4.44$ (s, 2H, $\left.\mathrm{CH}_{2}\right), 7.33-8.04$ (m, 10H, 10ArH).

$N-(5-\{[(4,5-d i p h e n y l-4 H-1,2,4-t r i a z o l-3-y l)$ sulfanyl $] m e t h y l\}-$ 1,3,4-thiadiazol-2-yl)-N-allylacetamide (7b) Yield: $62.1 \%$, mp: $212-214{ }^{\circ} \mathrm{C}$ (dec.). Analysis for $\mathrm{C}_{22} \mathrm{H}_{20} \mathrm{~N}_{6} \mathrm{OS}_{2}$ (448.56); calculated: C, 58.91; H, 4.49; N, 18.74; S, 14.30; found: C, 58.94; H, 4.51; N, 18.76; S, 
14.28. IR ( $\mathrm{KBr}), v\left(\mathrm{~cm}^{-1}\right): 3122(\mathrm{CH}$ aromatic), 2978, 1492, 742 (CH aliphatic), $1708(\mathrm{C}=\mathrm{O}), 1614(\mathrm{C}=\mathrm{N}), 1515$ $(\mathrm{C}-\mathrm{N}), 688(\mathrm{C}-\mathrm{S}) .{ }^{1} \mathrm{H}$ NMR (DMSO- $\left.d_{6}\right) \delta(\mathrm{ppm}): 2.11(\mathrm{~s}$, $\left.3 \mathrm{H}, \mathrm{CH}_{3}\right), 4.27\left(\mathrm{~s}, 2 \mathrm{H}, \mathrm{CH}_{2}\right), 4.35\left(\mathrm{~d}, J=5 \mathrm{~Hz}, 2 \mathrm{H}, \mathrm{CH}_{2}\right)$, $5.14-5.18\left(\mathrm{dd}, J=5 \mathrm{~Hz}, J=5 \mathrm{~Hz}, 2 \mathrm{H},=\mathrm{CH}_{2}\right), 5.81-5.86$ (m, 1H, CH), 7.34-8.07 (m, 10H, 10ArH).

$N-(5-\{[(4,5-d i p h e n y l-4 H-1,2,4-t r i a z o l-3-y l)$ sulfanyl $]$ methyl $\}-$ 1,3,4-thiadiazol-2-yl)-N-cyclohexylacetamide (7c) Yield: $87.5 \%$, mp: $193-195{ }^{\circ} \mathrm{C}$ (dec.). Analysis for $\mathrm{C}_{25} \mathrm{H}_{26} \mathrm{~N}_{6} \mathrm{OS}_{2}$ (490.64); calculated: C, 61.20; H, 5.34; N, 17.13; S, 13.07; found: C, 61.22; H, 5.32; N, 17.16; S, 13.05. IR (KBr), $v\left(\mathrm{~cm}^{-1}\right): 3108(\mathrm{CH}$ aromatic $), 2988,1487,755(\mathrm{CH}$ aliphatic), $1705(\mathrm{C}=\mathrm{O}), 1603(\mathrm{C}=\mathrm{N}), 1506(\mathrm{C}-\mathrm{N}), 674(\mathrm{C}-\mathrm{S})$. ${ }^{1} \mathrm{H}$ NMR (DMSO- $\left.d_{6}\right) \delta(\mathrm{ppm}): 1.36-1.84\left(\mathrm{~m}, 10 \mathrm{H}, 5 \mathrm{CH}_{2}\right.$ cyclohexane), $2.14\left(\mathrm{~s}, 3 \mathrm{H}, \mathrm{CH}_{3}\right), 3.64(\mathrm{~m}, 1 \mathrm{H}, \mathrm{CH}$ cyclohexane), 4.26 (s, 2H, $\mathrm{CH}_{2}$ ), 7.33-8.05 (m, 10H, 10ArH).

\section{$N-(5-\{[(4,5-d i p h e n y l-4 H-1,2,4-t r i a z o l-3-y l)$ sulfanyl $]$} methyl\}-1,3,4-thiadiazol-2-yl)-N-phenylacetamide

Yield: $67.7 \%$, mp: 209-211 ${ }^{\circ} \mathrm{C}$ (dec.). Analysis for $\mathrm{C}_{25} \mathrm{H}_{20} \mathrm{~N}_{6} \mathrm{OS}_{2}$ (484.59); calculated: C, 61.96; H, 4.16; N, 17.34; S, 13.23; found: C, 61.95; H, 4.08; N, 17.31; S, 13.26. IR (KBr), $v\left(\mathrm{~cm}^{-1}\right): 3098(\mathrm{CH}$ aromatic), $2978(\mathrm{CH}$ aliphatic), $1699(\mathrm{C}=\mathrm{O}), 1602(\mathrm{C}=\mathrm{N}), 1509(\mathrm{C}-\mathrm{N}), 694(\mathrm{C}-$ S). ${ }^{1} \mathrm{H}$ NMR (DMSO-d $) \delta$ (ppm): 2.12 (s, 3H, $\left.\mathrm{CH}_{3}\right), 4.22$ (s, 2H, $\mathrm{CH}_{2}$ ), 7.16-7.92 (m, 15H, 15ArH).

\section{$N-(5-\{[(4,5-$ diphenyl-4H-1,2,4-triazol-3-yl)sulfanyl $]$} methyl\}-1,3,4-thiadiazol-2-yl)-N-(4-bromophenyl)acetamide (7e) Yield: $84.6 \%$, mp: $222-224{ }^{\circ} \mathrm{C}$ (dec.). Analysis for $\mathrm{C}_{25} \mathrm{H}_{19} \mathrm{BrN}_{6} \mathrm{OS}_{2}$ (563.49); calculated: C, 53.29; H, 3.40; N, 14.91; S, 11.38; Br, 14.18; found: C, 53.33; H, 3.38; N, 14.95; S, 11.36. IR (KBr), $v\left(\mathrm{~cm}^{-1}\right): 3123$ ( $\mathrm{CH}$ aromatic), 2974, 1467 (CH aliphatic), 1712 (C=O), $1621(\mathrm{C}=\mathrm{N}), 1509$ $(\mathrm{C}-\mathrm{N}), 684(\mathrm{C}-\mathrm{S}) .{ }^{1} \mathrm{H}$ NMR (DMSO- $\left.d_{6}\right) \delta(\mathrm{ppm}): 2.15(\mathrm{~s}$, $\left.3 \mathrm{H}, \mathrm{CH}_{3}\right), 4.25$ (s, 2H, $\left.\mathrm{CH}_{2}\right), 7.27-7.94(\mathrm{~m}, 14 \mathrm{H}, 14 \mathrm{ArH})$.

\section{$N$-(5-\{[(4,5-diphenyl-4H-1,2,4-triazol-3-yl)sulfanyl]}

methyl\}-1,3,4-thiadiazol-2-yl)-N-(4-chlorophenyl)acetamide (7f) Yield: $59.8 \%$, mp: $229-231{ }^{\circ} \mathrm{C}$ (dec.). Analysis for $\mathrm{C}_{25} \mathrm{H}_{19} \mathrm{ClN}_{6} \mathrm{OS}_{2}$ (519.04); calculated: C, 57.85; H, 3.69; N, 16.19; S, 12.36; Cl, 6.83; found: C, 57.81; H, 3.65; N, 16.22; S, 12.37. IR ( $\mathrm{KBr}), v\left(\mathrm{~cm}^{-1}\right): 3090(\mathrm{CH}$ aromatic), 2980, 1451 ( $\mathrm{CH}$ aliphatic), $1695(\mathrm{C}=\mathrm{O}), 1601(\mathrm{C}=\mathrm{N}), 1521$ $(\mathrm{C}-\mathrm{N}), 689(\mathrm{C}-\mathrm{S}) .{ }^{1} \mathrm{H}$ NMR (DMSO- $\left.d_{6}\right) \delta(\mathrm{ppm}): 2.15(\mathrm{~s}$, $\left.3 \mathrm{H}, \mathrm{CH}_{3}\right), 4.24$ (s, 2H, $\left.\mathrm{CH}_{2}\right), 7.26-7.91$ (m, 14H, 14ArH).

\section{$N-(5-\{[(4,5-d i p h e n y l-4 H-1,2,4-t r i a z o l-3-y l)$ sulfanyl $]$} methyl)-1,3,4-thiadiazol-2-yl)-N-(4-methoxyphenyl)acetamide (7g) Yield: $62.8 \%$, mp: $174-176{ }^{\circ} \mathrm{C}$ (dec.). Analysis for $\mathrm{C}_{26} \mathrm{H}_{22} \mathrm{~N}_{6} \mathrm{O}_{2} \mathrm{~S}_{2}$ (514.62); calculated: C, 60.68; H, 4.31; N, 16.33; S, 12.46; found: C, 60.64; H, 4.29; N, 16.37; S, 12.45. IR $(\mathrm{KBr}), v\left(\mathrm{~cm}^{-1}\right): 3067(\mathrm{CH}$ aromatic), 2987, $1452(\mathrm{CH}$ aliphatic), $1710(\mathrm{C}=\mathrm{O}), 1611(\mathrm{C}=\mathrm{N}), 1508(\mathrm{C}-$ $\mathrm{N}), 679(\mathrm{C}-\mathrm{S}) .{ }^{1} \mathrm{H}$ NMR (DMSO-d $) \delta(\mathrm{ppm}): 2.09$ (s, 3H, $\left.\mathrm{CH}_{3}\right), 3.78\left(\mathrm{~s}, 3 \mathrm{H}, \mathrm{CH}_{3}\right), 3.87\left(\mathrm{~s}, 2 \mathrm{H}, \mathrm{CH}_{2}\right), 7.09-8.50(\mathrm{~m}$, $14 \mathrm{H}, 14 \mathrm{ArH})$.

$N-(5-\{[(4,5-d i p h e n y l-4 H-1,2,4-t r i a z o l-3-y l)$ sulfanyl $] m e t h y l\}-$ 1,3,4-thiadiazol-2-yl)-N-benzylacetamide (7h) Yield: $73.4 \%$, mp: $156-158{ }^{\circ} \mathrm{C}$ (dec.). Analysis for $\mathrm{C}_{26} \mathrm{H}_{22} \mathrm{~N}_{6} \mathrm{OS}_{2}$ (498.62); calculated: C, 62.63; H, 4.45; N, 16.85; S, 12.86; found: C, 62.67; H, 4.48; N, 16.81; S, 12.84. IR (KBr), v $\left(\mathrm{cm}^{-1}\right)$ : 3076 (CH aromatic), 2965, 1468 (CH aliphatic), $1713(\mathrm{C}=\mathrm{O}), 1614(\mathrm{C}=\mathrm{N}), 1523(\mathrm{C}-\mathrm{N}), 695(\mathrm{C}-\mathrm{S}) .{ }^{1} \mathrm{H}$ NMR $\left(\mathrm{DMSO}-d_{6}\right) \delta$ (ppm): 2.06 (s, 3H, $\left.\mathrm{CH}_{3}\right), 4.26\left(\mathrm{~s}, 2 \mathrm{H}, \mathrm{CH}_{2}\right)$, $4.75\left(\mathrm{~s}, 2 \mathrm{H}, \mathrm{CH}_{2}\right), 7.19-8.36(\mathrm{~m}, 15 \mathrm{H}, 15 \mathrm{ArH})$.

$N-(5-\{[(4,5-d i p h e n y l-4 H-1,2,4-t r i a z o l-3-y l)$ sulfanyl $] m e t h y l\}-$ 1,3,4-thiadiazol-2-yl)-N-(4-methoxybenzyl)acetamide (7i) Yield: $69.4 \%$, mp: $201-203{ }^{\circ} \mathrm{C}$ (dec.). Analysis for $\mathrm{C}_{27} \mathrm{H}_{24} \mathrm{~N}_{6} \mathrm{O}_{2} \mathrm{~S}_{2}$ (528.65); calculated: $\mathrm{C}, 61.34 ; \mathrm{H}, 4.58 ; \mathrm{N}$, 15.90; S, 12.13; found: C, 61.37; H, 4.59; N, 15.89; S, 12.16. IR $(\mathrm{KBr}), v\left(\mathrm{~cm}^{-1}\right): 3103(\mathrm{CH}$ aromatic), 2967, 1461, 756 ( $\mathrm{CH}$ aliphatic), $1704(\mathrm{C}=\mathrm{O}), 1607(\mathrm{C}=\mathrm{N}), 1514$ $(\mathrm{C}-\mathrm{N}), 697(\mathrm{C}-\mathrm{S}) .{ }^{1} \mathrm{H}$ NMR (DMSO-d $) \delta(\mathrm{ppm}): 2.16(\mathrm{~s}$, $\left.3 \mathrm{H}, \mathrm{CH}_{3}\right), 3.77$ (s, 3H, $\left.\mathrm{CH}_{3}\right), 4.24\left(\mathrm{~s}, 2 \mathrm{H}, \mathrm{CH}_{2}\right), 4.31$ (s, $\left.2 \mathrm{H}, \mathrm{CH}_{2}\right), 6.88-7.71(\mathrm{~m}, 14 \mathrm{H}, 14 \mathrm{ArH})$.

[(4,5-Diphenyl-4H-1,2,4-triazol-3-yl)sulfanyl] acetic acid (8) Compound $\mathbf{8}$ was obtained using the same method as described earlier for derivatives $\mathbf{5 a}-\mathbf{i}$. That is, a mixture of thiosemicarbazide $\mathbf{4 j}$ (10 mmol) and $20 \mathrm{~mL}$ of $2 \%$ aqueous solution of sodium hydroxide was refluxed for $2 \mathrm{~h}$. Then, the solution was neutralized with diluted hydrochloric acid and the formed precipitate was filtered and crystallized from ethanol.

Yield: $70.3 \%$, mp: $248-249{ }^{\circ} \mathrm{C}$ (dec.). Analysis for $\mathrm{C}_{16} \mathrm{H}_{13} \mathrm{~N}_{3} \mathrm{O}_{2} \mathrm{~S}$ (311.36); calculated: $\mathrm{C}, 61.72 ; \mathrm{H}, 4.21 ; \mathrm{N}$, 13.49; S, 10.30; found: C, 61.59; H, 4.19; N, 13.54; S, 10.28. IR (KBr), $v\left(\mathrm{~cm}^{-1}\right): 3079(\mathrm{CH}$ aromatic), 3045 (OH), 2982 (CH aliphatic), $1702(\mathrm{C}=\mathrm{O}), 1599(\mathrm{C}=\mathrm{N}), 688$ $(\mathrm{C}-\mathrm{S}) .{ }^{1} \mathrm{H}$ NMR (DMSO- $\left.d_{6}\right) \delta$ (ppm): 4.04 (s, 2H, $\mathrm{CH}_{2}$ ), 7.28-7.61 (m, 10H, 10ArH), 12.97 (s, 1H, OH).

4-Carboxymethyl-5-\{[(4,5-diphenyl-4H-1,2,4-triazol-3-yl) sulfanyl]methyl\}-4H-1,2,4-triazole-3(2H)-thione (9) Com -pound 9 was obtained using the same method as described earlier for derivatives $\mathbf{5 a} \mathbf{a}-\mathbf{i}$. That is, a mixture of thiosemicarbazide $4 \mathbf{k}(10 \mathrm{mmol})$ and $20 \mathrm{~mL}$ of $2 \%$ aqueous solution of sodium hydroxide was refluxed for $2 \mathrm{~h}$. Then, the solution 
was neutralized with diluted hydrochloric acid and the formed precipitate was filtered and crystallized from ethanol.

Yield: $97.2 \%$, mp: $157-159{ }^{\circ} \mathrm{C}$ (dec.). Analysis for $\mathrm{C}_{19} \mathrm{H}_{16} \mathrm{~N}_{6} \mathrm{O}_{2} \mathrm{~S}_{2}$ (424.50); calculated: C, 53.76; H, 3.80; N, 19.80; S, 15.11; found: C, 53.88; H, 3.81; N, 19.74; S, 15.47. IR (KBr), $v\left(\mathrm{~cm}^{-1}\right): 3228(\mathrm{NH}), 3095(\mathrm{OH}), 3062$ (CH aromatic), 2991 ( $\mathrm{CH}$ aliphatic), 1713 (C=O), 1605 $(\mathrm{C}=\mathrm{N}), 1504(\mathrm{C}-\mathrm{N}), 1343(\mathrm{C}=\mathrm{S}), 681(\mathrm{C}-\mathrm{S}) .{ }^{1} \mathrm{H}$ NMR $\left(\mathrm{DMSO}-d_{6}\right) \delta$ (ppm): 4.42 (s, 2H, $\left.\mathrm{CH}_{2}\right), 4.78$ (s, 2H, $\mathrm{CH}_{2}$ ), 7.27-7.56 (m, 10H, 10ArH), 13.80 (s, 1H, OH), 14.13 (brs, $1 \mathrm{H}, \mathrm{NH})$.

5-\{[(4,5-Diphenyl-4H-1,2,4-triazol-3-yl)sulfanyl]methyl\}2,5-dihydro-4H-1,2,4-triazole-3(2H)-thione (10) Compound 10 was obtained using the same method as described earlier for derivatives $\mathbf{5 a}-\mathbf{i}$. That is, a mixture of thiosemicarbazide $4 \mathbf{l}(10 \mathrm{mmol})$ and $20 \mathrm{~mL}$ of $2 \%$ aqueous solution of sodium hydroxide was refluxed for $2 \mathrm{~h}$. Then, the solution was neutralized with diluted hydrochloric acid and the formed precipitate was filtered and crystallized from ethanol.

Yield: $78.9 \%, \mathrm{mp}: 210-212{ }^{\circ} \mathrm{C}$ (dec.). Analysis for $\mathrm{C}_{17} \mathrm{H}_{14} \mathrm{~N}_{6} \mathrm{~S}_{2}$ (366.46); calculated: C, 55.72; H, 3.85; N, 22.93; S, 17.50; found: C, 55.58; H, 3.83; N, 23.01; S, 17.46. IR $(\mathrm{KBr}), v\left(\mathrm{~cm}^{-1}\right): 3256(\mathrm{NH}), 3079(\mathrm{CH}$ aromatic), 2956, 1461 ( $\mathrm{CH}$ aliphatic), $1603(\mathrm{C}=\mathrm{N}), 1510(\mathrm{C}-$ $\mathrm{N}), 1329(\mathrm{C}=\mathrm{S}), 695(\mathrm{C}-\mathrm{S}) .{ }^{1} \mathrm{H}$ NMR (DMSO- $\left.d_{6}\right) \delta(\mathrm{ppm})$ : 4.04 (s, 2H, $\mathrm{CH}_{2}$ ), 7.29-7.92 (m, 10H, 10ArH), 13.33 (s, 1H, NH), 14.15 (brs, 1H, NH).

[3-\{[(4,5-Diphenyl-4H-1,2,4-triazol-3-yl)sulfanyl]methyl\}1-(pyrrolidin-1-ylmethyl)-5-thioxo-1,5-dihydro-4H-1,2,4triazol-4-yl]acetic acid (11) To a solution of $10 \mathrm{mmol}$ of compound 9 in ethanol, pyrrolidine $(10 \mathrm{mmol})$ and formaldehyde $(0.2 \mathrm{~mL})$ were added. The mixture was stirred for $2 \mathrm{~h}$ at room temperature. After that, distilled water was added and the precipitate that formed was filtered, washed with distilled water, and crystallized from ethanol.

Yield: $66.8 \%$, mp: $173-175^{\circ} \mathrm{C}$ (dec.). Analysis for $\mathrm{C}_{24} \mathrm{H}_{25} \mathrm{~N}_{7} \mathrm{O}_{2} \mathrm{~S}_{2}$ (507.63); calculated: C, 56.78; H, 4.96; N, 19.31; S, 12.63; found: C, 56.80; H, 4.97; N, 19.34; S, 12.66. IR (KBr), $v\left(\mathrm{~cm}^{-1}\right): 3100(\mathrm{OH}), 3069(\mathrm{CH}$ aromatic), 2962 ( $\mathrm{CH}$ aliphatic), $1715(\mathrm{C}=\mathrm{O}), 1611(\mathrm{C}=\mathrm{N})$, $1514(\mathrm{C}-\mathrm{N}), 1367(\mathrm{C}=\mathrm{S}), 692(\mathrm{C}-\mathrm{S}) .{ }^{1} \mathrm{H}$ NMR (DMSO-d $\left.{ }_{6}\right)$ $\delta$ (ppm): 1.66-1.72 (m, 4H, 2CH $\mathrm{CH}_{2}, 2.29(\mathrm{t}, J=5 \mathrm{~Hz}, 2 \mathrm{H}$, $\left.\mathrm{CH}_{2}\right), 2.68\left(\mathrm{t}, J=5 \mathrm{~Hz}, 2 \mathrm{H}, \mathrm{CH}_{2}\right), 4.27\left(\mathrm{~s}, 2 \mathrm{H}, \mathrm{CH}_{2}\right), 4.58$ $\left(\mathrm{s}, 2 \mathrm{H}, \mathrm{CH}_{2}\right), 4.69\left(\mathrm{~s}, 2 \mathrm{H}, \mathrm{CH}_{2}\right), 7.47-8.08(\mathrm{~m}, 10 \mathrm{H}$, 10ArH), 13.68 (s, 1H, OH).

5-\{[(4,5-Diphenyl-4H-1,2,4-triazol-3-yl)sulfanyl $]$ methyl $\}$ 2-(pyrrolidin-1-ylmethyl)-2,4-dihyro-3H-1,2,4-triazole-3thione (12) To a solution of $10 \mathrm{mmol}$ of compound $\mathbf{1 0}$ in ethanol, pyrrolidine $(10 \mathrm{mmol})$ and formaldehyde $(0.2 \mathrm{~mL})$ were added. The mixture was stirred for $2 \mathrm{~h}$ at room temperature. After that, distilled water was added and the precipitate that formed was filtered, washed with distilled water, and crystallized from ethanol.

Yield: $74.8 \%$, mp: $224-226{ }^{\circ} \mathrm{C}$ (dec.). Analysis for $\mathrm{C}_{22} \mathrm{H}_{23} \mathrm{~N}_{7} \mathrm{~S}_{2}$ (449.59); calculated: C, 58.77; H, 5.16; N, 21.81; S, 14.26; found: C, 58.79; H, 5.14; N, 21.83; S, 12.24. IR (KBr), $v\left(\mathrm{~cm}^{-1}\right): 3290(\mathrm{NH}), 3098(\mathrm{CH}$ aromatic), 2978, $1482(\mathrm{CH}$ aliphatic), $1623(\mathrm{C}=\mathrm{N}), 1522(\mathrm{C}-$ $\mathrm{N}), 1341(\mathrm{C}=\mathrm{S}), 685(\mathrm{C}-\mathrm{S}) .{ }^{1} \mathrm{H}$ NMR (DMSO-d $) \delta(\mathrm{ppm})$ : 1.67-1.73 (m, 4H, 2CH$), 2.32\left(\mathrm{t}, J=5 \mathrm{~Hz}, 2 \mathrm{H}, \mathrm{CH}_{2}\right)$, $2.77\left(\mathrm{t}, \mathrm{J}=5 \mathrm{~Hz}, 2 \mathrm{H}, \mathrm{CH}_{2}\right), 4.05\left(\mathrm{~s}, 2 \mathrm{H}, \mathrm{CH}_{2}\right), 4.68(\mathrm{~s}, 2 \mathrm{H}$, $\mathrm{CH}_{2}$ ), 7.36-8.35 (m, 10H, 10ArH), 14.68 (brs, 1H, NH).

\section{Microbiology}

\section{Materials and methods}

All synthesized compounds were preliminarily tested for their in vitro antibacterial activity against Gram-positive and -negative reference bacterial strains and next by the broth microdilution method against the selected bacterial strains.

Panel reference strains of aerobic bacteria from the American Type Culture Collection, including six Grampositive bacteria, S. aureus ATCC 25923, S. aureus ATCC 6538, S. epidermidis ATCC 12228, B. subtilis ATCC 6633, B. cereus ATCC 10876, M. luteus ATCC 10240, and four Gram-negative bacteria, Escherichia coli ATCC 25922, Klebsiella pneumoniae ATCC 13883, Proteus mirabilis ATCC 12453, Pseudomonas aeruginosa ATCC 9027, were used. Microbial suspensions with an optical density of 0.5 McFarland standard $150 \times 10^{6} \mathrm{CFU} / \mathrm{mL}$ (CFUs-colony forming units) were prepared in sterile $0.85 \% \mathrm{NaCl}$. All stock solutions of the tested compounds were prepared in DMSO. The medium with DMSO at the final concentration and without the tested compounds served as the controlno microbial growth inhibition was observed.

Preliminary antimicrobial potency in vitro of the tested compounds was screened using the agar dilution method on the basis of the bacterial growth inhibition on the MuellerHinton agar containing the compounds at a concentration of $1,000 \mu \mathrm{g} / \mathrm{mL}$. The plates were poured on the day of testing. $10 \mu \mathrm{L}$ of each bacterial suspension was put onto the prepared solid media. The plates were incubated at $37{ }^{\circ} \mathrm{C}$ for $18 \mathrm{~h}$ (Bourgeois et al., 2007).

The antibacterial activity in vitro of the potentially active compounds was determined by the broth microdilution method on the basis of MIC, usually defined as the lowest concentration of the compound at which there was no visible growth of microorganisms (White et al., 2002). Determination of the MIC value was achieved by the broth microdilution method according to a CLSI (Clinical and 
Laboratory Standards Institute) recommendation with some modifications (2008). The 96-well microplates were used; $198 \mu \mathrm{L}$ of Mueller-Hinton broth with a series of twofold dilutions of the tested compound in the range of the final concentrations from 0.24 to $1,000 \mu \mathrm{g} / \mathrm{mL}$ was inoculated with $2 \mu \mathrm{L}$ of microbial suspension (total volume per each well-200 $\mu \mathrm{L}$ ). After incubation (at $35^{\circ} \mathrm{C}$ for $18 \mathrm{~h}$ ), spectrophotometric measurements of optical density $\left(\mathrm{OD}_{600}\right)$ of the bacterial cultures with the tested compounds were performed in order to determine MIC. $\mathrm{OD}_{600}$ of bacterial cultures in the medium without the tested compounds was used as a control. The blank control wells with twofold dilution of each of the tested compounds added to the Mueller-Hinton broth without bacterial suspension were incubated under the same conditions. Cefuroxime, belonging to the second generation of cephalosporins, was used as a control antimicrobial agent.

Conflict of interest The authors declare no conflict of interest.

Open Access This article is distributed under the terms of the Creative Commons Attribution License which permits any use, distribution, and reproduction in any medium, provided the original author(s) and the source are credited.

\section{References}

Allen FH (2002) The Cambridge Structural Database: a quarter of million crystal structures and rising. Acta Crystallogr B 58:380388

Almasirad A, Tabatabai SA, Faizi M, Kebriaeezadeh A, Mehrabi N, Dalvandi A, Shafiee A (2004) Synthesis and anticonvulsant activity of new 2-substituted-5-[2-(2-fluorophenoxy)phenyl]-1, 3,4-oxadiazoles and 1,2,4-triazoles. Bioorg Med Chem Lett 14:6057-6059

Al-Soud YA, Al-Dweri MN, Al-Masoudi NA (2004) Synthesis, antitumor and antiviral properties of some 1,2,4-triazole derivatives. Farmaco 59:775-783

Bailey EM, Krakovsky DJ, Rybak M (1990) The triazole antifungal agents: a review of itraconazole and fluconazole. Pharmacotherapy $10: 146-153$

Bourgeois I, Pestel-Caron M, Lemeland JF, Pons JL, Caron F (2007) Tolerance to the glycopeptides vancomycin and teicoplanin in coagulase-negative Staphylococci. Antimicrob Agents Chemother 51(2):740-743

Clemons M, Coleman RE, Verma S (2004) Cancer Treat Rev 30: 325-332

CLSI (2008) Performance standards for antimicrobial susceptibility testing; Eighteenth International Supplement. CLSI document M7-MIC. Clinical Laboratory Standards Institute, Wayne

Collin X, Sauleau A, Coulon J (2003) 1,2,4-Triazolo mercapto and aminonitriles as potent antifungal agents. Bioorg Med Chem Lett 13:2601-2605

Demirbas A, Sahin D, Demirbas N, Karaoglu SA (2009) Synthesis of some new 1,3,4-thiadiazol-2-ylmethyl-1,2,4-triazole derivatives and investigation of their antimicrobial activities. Eur $\mathrm{J}$ Med Chem 44:2896-2903

Dobosz M, Pachuta-Stec A (1995) Cyclization of 1-cyanoacetyl-4substituted thiosemicarbazides to 1,2,4-triazole or 1,3,4-thiadiazole derivatives. Acta Pol Pharm 52:103-111
Dobosz M, Pachuta-Stec A (1996) Synthesis of new derivatives of 3-benzyl- $\Delta^{2}$-1,2,4-triazoline-5-thione and 5-benzyl-1,3,4-thiadiazole. Acta Pol Pharm 53:123-131

Dobosz M, Pitucha M, Wujec M (1996) The reactions of cyclization of thiosemicarbazide derivatives to 1,2,4-triazole or 1,3,4thiadiazole system. Acta Pol Pharm 53:31-38

Dogan HN, Duran A, Rollas S, Sener G, Uysal MK, Gülen D (2002) Synthesis of new 2,5-disubstituted-1,3,4-thiadiazoles and preliminary evaluation of anticonvulsant and antimicrobial activities. Bioorg Med Chem 10:2893-2898

Duran A, Dogan HN, Rollas S (2002) Synthesis and preliminary anticancer activity of new 1,4-dihydro-3-(3-hydroxy-2-naphthyl)-4-substituted-5H-1,2,4-triazoline-5-thiones. Farmaco 57:559-564

El Shehry MF, Abu-Hashem AA, El-Telbani EM (2010) Synthesis of 3-((2,4-dichlorophenoxy)methyl)-1,2,4-triazolo(thiadiazoles and thiadiazines) as anti-inflammatory and molluscicidal agents. Eur J Med Chem 45:1906-1911

Gadad AK, Noolvi MN, Karpoormath RV (2004) Synthesis and antitubercular activity of a series of 2-sulfonamido/trifluoromethyl6-substituted imidazo-[2,1- $b]-1,3,4$-thiadiazole derivatives. Bioorg Med Chem 12:5651-5659

Gülerman NN, Doğan HN, Rollas S, Johansson C, Çelik C (2001) Synthesis and structure elucidation of some new thioether derivatives of 1,2,4-triazoline-3-thiones and their antimicrobial activities. Farmaco 56:953-958

Harish K, Sadique AJ, Suroor AK, Mohammad A (2008) 1,3,4Oxadiazole/thiadiazole and 1,2,4-triazole derivatives of biphenyl-4-yloxy acetic acid: synthesis and preliminary evaluation of biological properties. Eur J Med Chem 43:2688-2698

Holmwood G, Buechel KH, Plempel M, Haller J (1982) Antimicrobial azoles. Chem Abstr 96:62979s. Patent RFN DE 3018865, 1981

Kaplaushenko AH, Panasenko OI, Knish EH, Scherbina RO (2008) Synthesis, physicochemical and biological properties of 2-(5-R14-R2-1,2,4-triazol-3-ylthio)acetic acids. Farm Zh 2:67-72

Klimešová V, Zahajská L, Waisser K, Kaustová J, Möllmann U (2004) Synthesis and antimycobacterial activity of 1,2,4-triazole 3-benzylsulfanyl derivatives. Farmaco 59:279-288

Kumar D, Kumar NM, Chang K-H, Shah K (2010) Synthesis and anticancer activity of 5-(3-indolyl)-1,3,4-thiadiazoles. Eur J Med Chem 45:4664-4668

Liesen AP, De Aquino TM, Carvalho CS, Lima VT, De Araújo JM, De Lima JG, De Faria AR, De Melo EJT, Alves AJ, Alves EW, Alves AQ, Góes AJS (2010) Synthesis and evaluation of antiToxoplasma gondii and antimicrobial activities of thiosemicarbazides, 4-thiazolidinones and 1,3,4-thiadiazoles. Eur J Med Chem 45:3685-3691

McGinnis MR, Pasarell L, Sutton DA, Fothergill AW, Cooper CR, Rinaldi MG (1997) Antimicrob Agents Chemother 41: $1832-1834$

Oxford Diffraction, Xcalibur CCD System (2006) CrysAlis Software System, Version 1.171. Oxford Diffraction Ltd., Abingdon

Padmavathi V, Sudhakar Reddy G, Padmaja A, Kondaiah P, AliShazia (2009) Synthesis, antimicrobial and cytotoxic activities of 1,3,4-oxadiazoles, 1,3,4-thiadiazoles and 1,2,4-triazoles. Eur J Med Chem 44:2106-2112

Pick C (1997) Antinociceptive interaction between alprazolam and opioids. Brain Res Bull 42:239-243

Ramasubbu N, Parthasarathy R (1989) Short S...O contacts: structure of 2,5-bis( $p$-methoxyphenylhydroxymethyl)thiophene. Acta Crystallogr C 45:457-460

Schenone S, Brullo C, Bruno O, Bondavalli F, Ranise A, Filippelli W et al (2006) New 1,3,4-thiadiazole derivatives endowed with analgesic and anti-inflammatory activities. Bioorg Med Chem 14:1698-1705 
Sheldrick GM (2008) A short history of SHELX. Acta Crystallogr A 64:112-122

Shiradkar MR, Murahari KK, Gangadasu HR, Suresh T, Kalyan ChA, Panchal D, Kaur R, Burange P, Ghogare J, Mokale V, Raut M (2007) Synthesis of new $S$-derivatives of clubbed triazolyl thiazole as anti-Mycobacterium tuberculosis agents. Bioorg Med Chem 15:3997-4008

Shiroki M, Tahara T, Araki K (1976) Chem Abstr 84:59588k. Japanese Patent 75100096, 1975

Siwek A, Paneth P (2007) Computational studies of the cyclization of thiosemicarbazides. J Phys Org Chem 20:463-468

Siwek A, Wujec M, Dobosz M, Wawrzycka-Gorczyca I (2010) Study of direction of cyclization of 1-azolil-4-aryl/alkyl-thiosemicarbazides. Heteroat Chem 21(7):521-532

Turan-Zitouni G, Kaplancikli ZA, Erol K, Kiliç FS (1999) Synthesis and analgesic activity of some triazoles and triazolothiadiazines. Farmaco 54:218-223
Ulusoy N, Gürsoy A, Ötük G (2001) Synthesis and antimicrobial activity of some 1,2,4-triazole-3-mercaptoacetic acid derivatives. Farmaco 56:947-952

Wei Q-L, Zhang S-S, Gao J, Li W-H, Xu L-Z, Yu Z-G (2006) Synthesis and QSAR studies of novel triazole compounds containing thioamide as potential antifungal agents. Bioorg Med Chem 14:7146-7153

White EL, Suling WJ, Ross LJ, Seitz LE, Reynolds RC (2002) 2-Alkoxycarbonylaminopyridines: inhibitors of Mycobacterium tuberculosis FtsZ. J Antimicrob Chemother 50:111-114

Wilson AJC (ed) (1995) International tables for crystallography, vol C. Kluwer Academic Publishers, Dordrecht

Wujec M, Paneth P (2007) Mechanism of 4-methyl-1,2,4-triazol-3thiole reaction with formaldehyde. A DFT study. J Phys Org Chem 20:1043-1049 